\title{
Environmental Releases for Calendar Year 1997
}

B. P. Gleckler

Waste Management Federal Services of Hanford, Inc.

Date

August 1998

Prepared for the U.S. Department of Energy Assistant Secretary for Environmental Management

FUOR DANEL "WANORD,

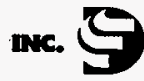

Richland, Washington 


\section{RELEASE AUTHORIZATION}

$$
\text { Document Number: HNF-EP-0527-7 }
$$

Document Title:

Environmental Releases for Calendar Year 1997

This document, reviewed in accordance with DOE Order 1430.1D, "Scientific and Technical Information Management," and DOE G 1430.1D-1, "Guide to the Management of Scientific and Technical Information," does not contain classified or sensitive unclassified information and is:

\section{APPROVED FOR PUBLIC RELEASE}

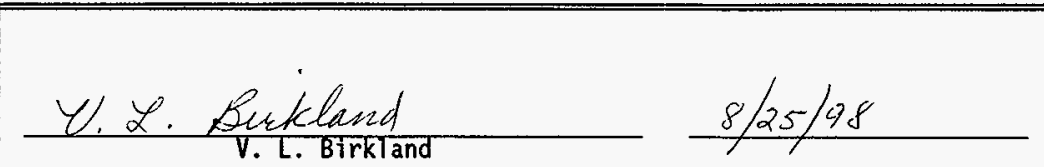

Lockheed Mart in Services, Inc.

Document Control/Information Clearance

hoviewed tor Appliod Technology, Bubineti Sonsitive, Classifiod, Copyrighted, Export Controlied, Patent, Personel/Private, Propietany, Protected CRADA. Trademark, Unclassified Controlled Nucloar Information.

Trademark Disclaimer. Reference heroin to any specific commorcial product, process, or sorvico by trade name, trademark, manufacturot, or otherwise, does not nocestarily constizute or imply its andersoment, recommondation, or favoring by the Unitod States Government or any agency thereof or its contractors or subeontractors. The viows and opinions of authore oxprostod heroin do not nocossarily state or roflect those of the United States Government or any agency thereof. This report has been reproduced from the beet available copy.

Printed in tho United States of America.

Avallable to the U.S. Department of Energy and its contractors trom the U.S. Depertment of Energy Office of Scientific and Technical Information, P.O. Box 62, Oak Ridge, TN 37831; Tolephono: 423/576-8401.

Avoilable to the public from the U.S. Department of Commerce National Technical Information Service, 5285 Port Royal Road, Springfield, VA 22161: Telophone: 703/487-4650. 
LEGAL DISCLAIMER

This report was propered as an account of work sponsored by an agancy of tho United States Government. Neither the United States Government nor any agency thereof, nor any of their omployese, nor any of their contractors, subcontractors or their omployess, makes any warranty, express or implied, or aseumes any legal liabitity or responsibility for the accuracy, completeness, or any third party's use or the results of such use of any information, apparatus, product, or process disclosed, or represents that its use would not infringe privately owned rights. Reference herein to any specific commercial product, process, or service by trade name, trademark, menufacturer, or otherwise, does not nocessarijy constitute or imply its endorsement, recommendetion, of favoring by the Unitod States Government or any egency thereof or its contractors or subcontractors. The views and opinions of authors expressed herein do not necessarily state of reflect those of the United States Government or any agency thereof.

This report has been reproduced from the best available copy.

Available in paper copy and microfiche.

Avaisable to the U.S. Department of Energy

and its contractors from

U.S. Depertment of Energy

Office of Scientific and Tochnical Information (OSTI)

P.O. Box 62

Oak Ridge, TN 37831

(615) 576-8401

Available to the public from the U.S. Department of Commerce

National Tochnical Information Sorvice (NTIS)

5285 Port Royal Road

Springfield, VA 22161

(703) $487-4650$

Printed in the United States of Americe

DISCLM-1.CHP (8-95) 
HNF-EP-0527-7

\section{APPROVAL PAGE}

Document Title: Environmental Releases for Calendar Year 1997

Prepared by:

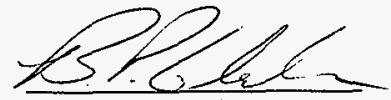

B. P. Gleckler, Author

$$
\frac{8 / 24 / 98}{\text { Date }}
$$

Air \& Water Services

Waste Management Federal Services of Hanford, Inc.

Approved by:

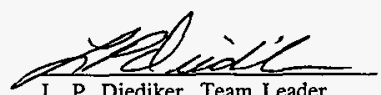

L. P. Diediker, Team Leader

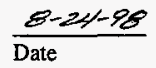

Air \& Water Services

Waste Management Federal Services of Hanford, Inc.

Approved by: $\frac{\text { EriM.Greagn }}{\text { E. M. Greager, Managet }} \frac{8 / 24 / 98}{\text { Date }}$ Air \& Water Services

Waste Management Federal Services of Hanford, Inc.

Approved by:
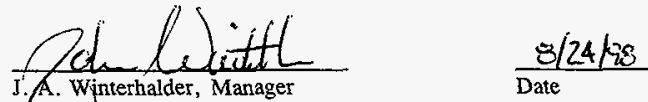

fuviropmental Services

Waste Management Federal Services of Hanford, Inc.

Approved by:

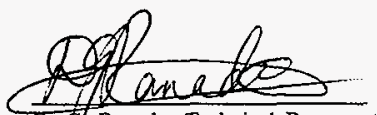

D. Ranade, Technical Representative

Environmental Integration

Fluor Daniel Hanford, Inc.
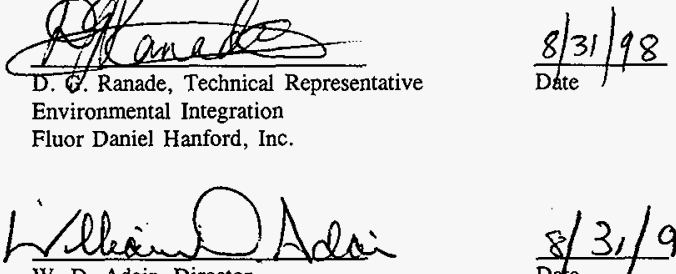

W. D. Adair, Director

Environmental Protection

$\frac{8 / 3 / 98}{02 / e}$

Fluor Daniel Hanford, Inc. 


\section{HNF-EP-0527-7}

This page intentionally left blank. 
HNF-EP-0527-7

\section{EXECUTIVE SUMMARY}

This report fulfills the annual environmental release reporting requirements of U.S. Department of Energy (DOE) Order 5400.1. This report provides supplemental information to the Hanford Site Environmental Report (PNNL-11795). The Hanford Site Environmental Report provides an update on the environmental status of the Hanford Site. The sitewide annual report summarizes the degree of compliance with applicable environmental regulations and informs the public concerning the impact of Hanford Site operations on the surrounding environment.

Like the Hanford Site Environmental Report, this annual report presents a summary of the environmental releases from facilities and activities managed by the Fluor Daniel Hanford, Incorporated (FDH), and Bechtel Hanford, Incorporated (BHI). In addition to the summary data, this report also includes detailed data on air emissions, liquid effluents, and hazardous substances released to the environment during calendar year 1997.

Comprehensive data summaries of air emissions and liquid effluents in 1997 are displayed in Tables ES-1 through ES-5. These tables represent the following:

- Table ES-1. Radionuclide air emissions data (detailed data on emissions are . presented in Section 2.0)

- Table ES-2. Data on radioactive liquid effluents discharged to the soil (detailed data are presented in Section 3.0)

- Table ES-3. Radionuclides discharged to the Columbia River (detailed data are presented in Section 3.0)

- Table ES-4. Nonradioactive air emissions data (detailed data are presented in Section 2.0)

- Table ES-5. Total Volumes and Flow Rates of 200/600 Area Radioactive Liquid Effluents (detailed data are presented in Section 3.0). 


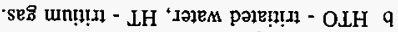

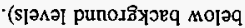

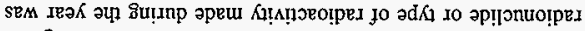

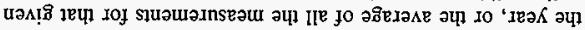

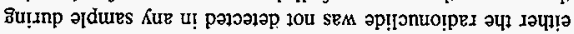

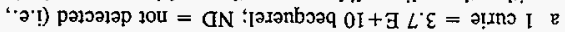
:

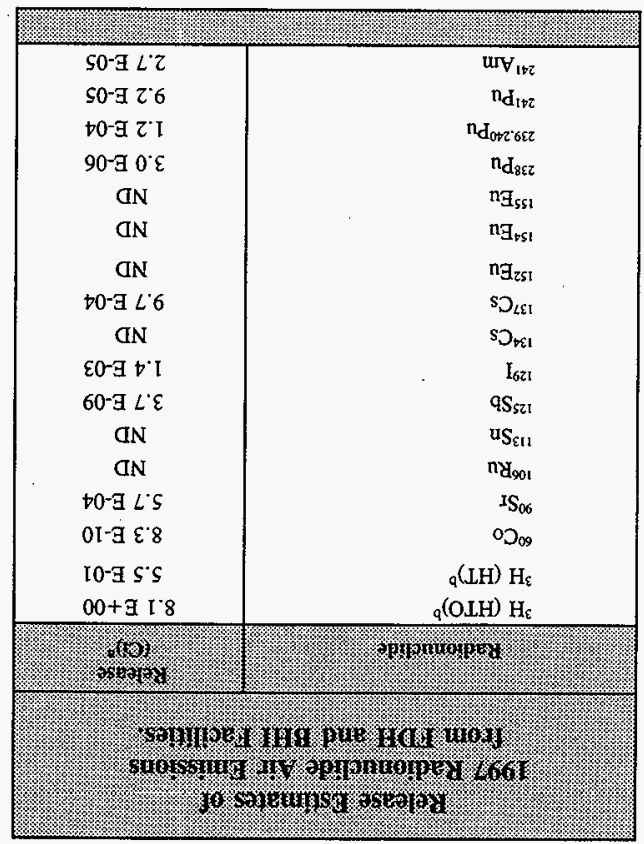

I-SH PIqEI 


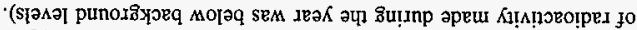

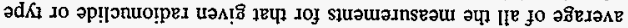

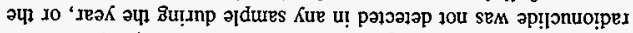

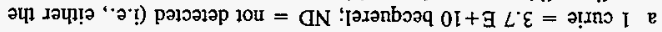
:aroN

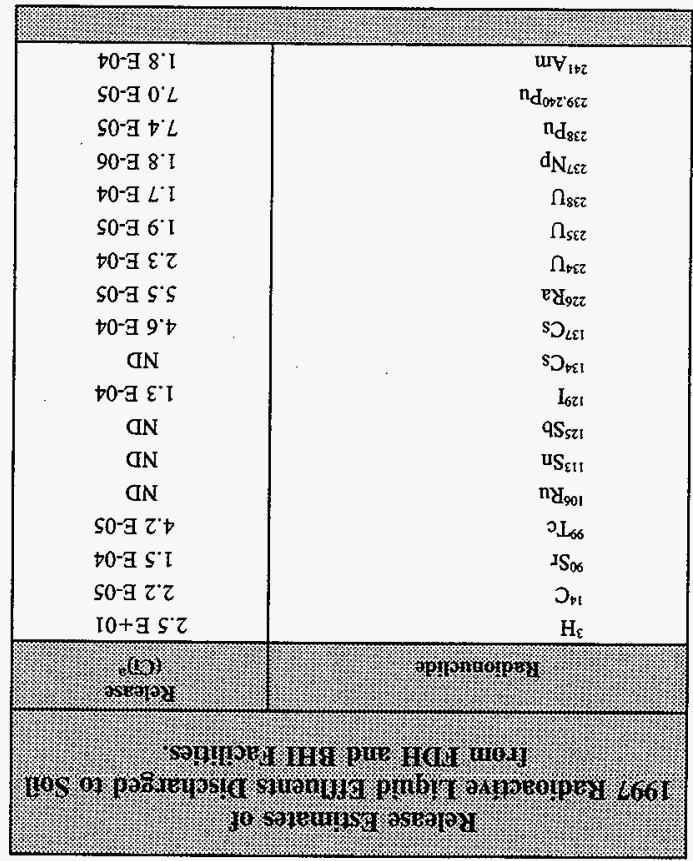

z-SG ә[q] 


\section{Table ES-3}

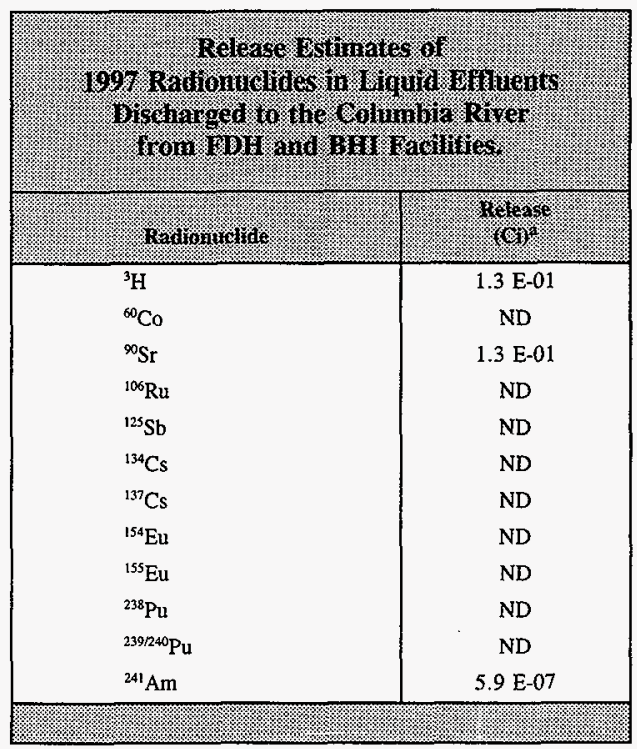

Note:

a 1 curie $=3.7 \mathrm{E}+10$ becquerel; $\mathrm{ND}=$ not detected (i.e., either the radionuclide was not detected in any sample during the year, or the average of all the measurements for that given radionuclide or type of radioactivity made during the year was below background levels). 
Table ES-4

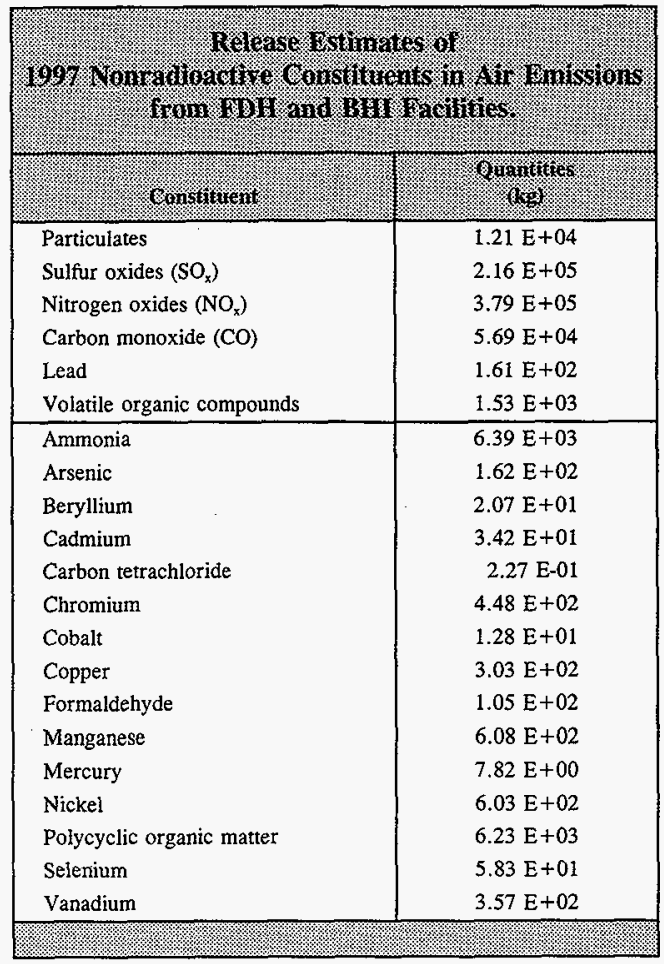


Table ES-5

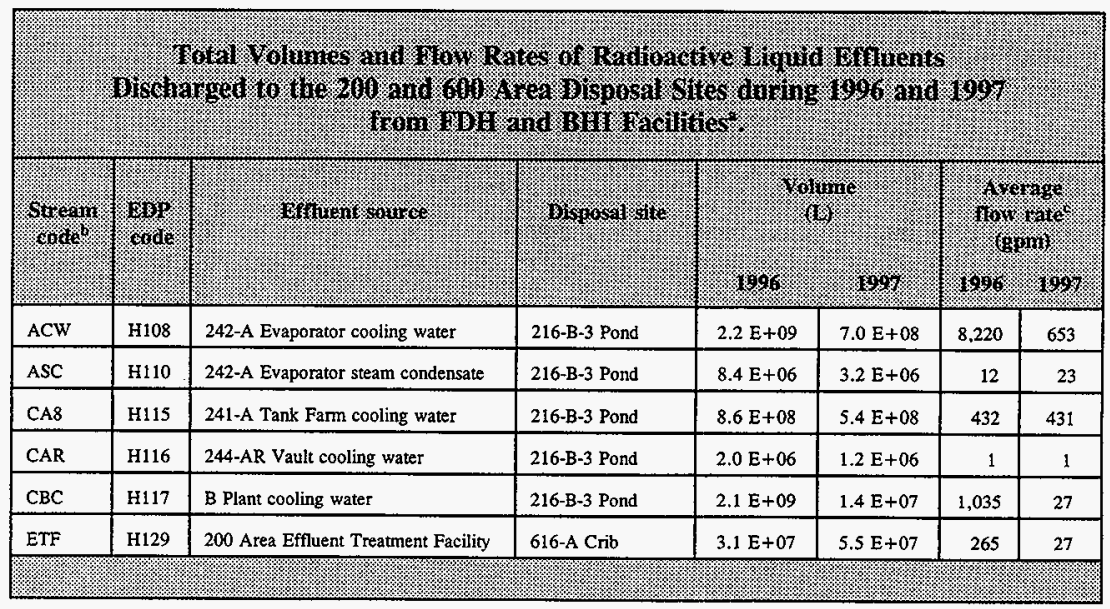

Notes:

a These discharges do not include discharges to the 200 East Area Treated Effluent Disposal Facility, because these discharges meet drinking water standards. Currenty, BHI does not manage any facilities that discharge radioactive liquid effluents to the 200 and 600 Areas.

b Stream codes are alpha numeric designators for specific liquid efftuent sources.

c Average flow rate for each discharge and/or sampling period, $1 \mathrm{gpm}=3.785 \mathrm{Lpm}$. 


\section{CONTENTS}

1.0 INTRODUCTION $\ldots \ldots \ldots \ldots \ldots \ldots \ldots \ldots \ldots \ldots \ldots \ldots$ 1-1

1.1 TYPES AND LOCATIONS OF RELEASES $\ldots \ldots \ldots \ldots \ldots \ldots$. . . . . .

1.2 ENVIRONMENTAL RELEASE LIMITS AND GUIDELINES . . . . . . . 1-2

1.2.1 Limits for Radioactive Releases . . . . . . . . . . . . . 1-2

1.2.2 Limits for Nonradioactive Releases $\ldots \ldots \ldots \ldots \ldots \ldots$ 1-3

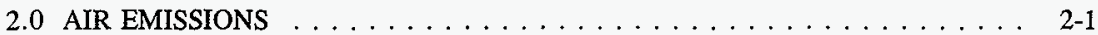

2.1 RADIONUCLIDE AIR EMISSIONS $\ldots \ldots \ldots \ldots \ldots \ldots \ldots \ldots \ldots . \ldots \ldots$

2.1.1 Mitigation of Radionuclide Air Emissions . . . . . . . . . . . 2-1

2.1.2 Radionuclide Air Emissions Data ... . . . . . . . . . . . 2-2

2.2 NONRADIOACTIVE AIR EMISSIONS $\ldots \ldots \ldots \ldots \ldots \ldots . \ldots \ldots$ 2-2

3.0 LIQUID EFFLUENTS $\ldots \ldots \ldots \ldots \ldots \ldots \ldots \ldots \ldots \ldots \ldots$. $\ldots \ldots \ldots$

3.1 NPDES PERMITTED DISCHARGES TO COLUMBIA RIVER . . . . . . . 3-1

3.1 .1 1908-K Outfall . . . . . . . . . . . . . . . . . . . . . . . 3- 3-2

3.1 .2 N-Springs . . . . . . . . . . . . . . . . 3-2

3.1 .3300 Area TEDF . . . . . . . . . . . . . . . 3-2

3.2 STATE PERMITTED DISCHARGES TO THE SOIL . . . . . . . . 3-2

3.2 .1200 Area TEDF . . . . . . . . . . . . . . . . . . 3 3-3

3.2 .2200 Area ETF . . . . . . . . . . . . . . . . . . . 3-3

3.2.3 400 Area Secondary Cooling Water $\ldots \ldots \ldots \ldots$. . . . . . . 3-3

3.2.4 183-N Backwash Discharge Pond . . . . . . . . . . . . . . 3-3

$3.2 .5100-\mathrm{N}$ Sewage Lagoon . . . . . . . . . . . . . . . . . . . . . . 3-3

3.2.6 Hydrotesting, Maintenance, and Construction Discharges . . . . . . . . 3-4

3.2.7 Cooling Water and Steam Condensate Discharges . . . . . . . . . . 3-4

3.2.8 Storm Water Discharges . . . . . . . . . . . . . . . . . . 3-4

3.3 SANITARY SEWAGE DISCHARGES TO THE SOIL $\ldots \ldots \ldots \ldots . . .4$

4.0 HAZARDOUS SUBSTANCE RELEASES $\ldots \ldots \ldots \ldots \ldots \ldots$. . . . . . . . . .

4.1 NONROUTINE RELEASES $\ldots \ldots \ldots \ldots \ldots \ldots \ldots \ldots$. . . . . . . .

4.2 ROUTINE CONTINUOUS RELEASES $\ldots \ldots \ldots \ldots \ldots$. . . . . . .

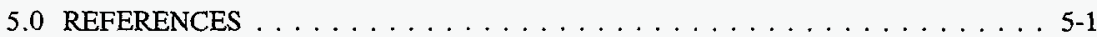




\section{LIST OF TABLES}

2-1 Release Estimates of 1997 Radionuclide Air Emissions from FDH and BHI Facilities . . . . . . . . . . . . . . 2-3

2-2 1997 Hanford Site Radionuclide Air Emissions Data for Major Point Sources from FDH and BHI . . . . . . . . . . . 2-4

2-3 1997 Hanford Site Radionuclide Air Emissions Data for Minor Point Sources from FDH and BHI . . . . . . . . . . . . 2-8

2-4 1997 Hanford Site Nonradioactive Air Emissions Data by Source . . . . . . . . 2-15

2-5 1997 Fuel Consumption from Powerhouse Boilers . . . . . . . . . . . . . 2-18

3-1 National Pollutant Discharge Elimination System (NPDES) and State Permitted Discharge Points . . . . . . . . . . . . . . 3-5

3-2 Summary of National Pollutant Discharge System (NPDES) Constituents for $1997 \ldots \ldots \ldots \ldots$. . . . . . . . . . . . . . . . .

3-3 1997 Radionuclide Liquid Effluent Data for Individual Effluent Streams Discharged to the Environment from FDH and BHI Facilities . . . . . 3-8

3-4 Summary of the 1997 Discharge Monitoring Reports for State Permitted Discharge Points . . . . . . . . . . . . . . . . . . . 3-11

3-5 Sanitary Sewage Discharged to the Soil in $1997 \ldots \ldots \ldots \ldots \ldots$. . . . . . . . . 


\section{GLOSSARY}

BHI Bechtel Hanford, Incorporated

CERCLA Comprehensive Environmental Response, Compensation, and Liability Act of 1980

CFR

DCG

DOE

Code of Federal Regulations

derived concentration guide

DOE-RL U.S. Department of Energy, Richland Operations Office

Ecology

EDE

EDP Code

State of Washington Department of Ecology

EP

effective dose equivalent

Electronic data processing code

EPA

external publication

ERDF

U.S. Environmental Protection Agency

ESPC

Environmental Restoration Disposal Facility

FDH

energy savings performance contract

FFTF

Fluor Daniel Hanford, Incorporated

HEPA

HT

Fast Flux Test Facility

high-efficiency particulate air (filter)

tritiated water

LWDF Liquid Waste Disposal Facility

MASF Maintenance and Storage Facility

MEI

mrem

maximally exposed individual

ND

millirem (unit of dose)

not detected

NPDES National Pollutant Discharge Elimination System

PHMC Project Hanford Management Contract

PFP Plutonium Finishing Plant

PSD Prevention of Significant Deterioration

PNNL Pacific Northwest National Laboratory

POTW publicly owned treatment works (city of Richland)

ppm parts per million

PUREX plutonium-uranium extraction

RCRA Resource Conservation and Recovery Act of 1976

REDOX Reduction-Oxidation

RQ reportable quantity

SALDS State-Approved Land Disposal Site

TEDF Treated Effluent Disposal Facility

TRIGA Test Reactor and Isotope Production, General Atomics

TRU transuranic (waste)

TRUSAF 224-T Transuranic Waste Storage and Assay Facility

$\mathrm{UO}_{3}$

uranium trioxide 
HNF-EP-0527-7

\section{GLOSSARY (continued)}

WAC Washington Administrative Code

WESF Waste Encapsulation Storage Facility

WDOH State of Washington Department of Health

WMH Waste Management Federal Services of Hanford, Incorporated

WSCF Waste Sampling and Characterization Facility 
HNF-EP-0527-7

\section{ENVIRONMENTAL RELEASES FOR CALENDAR YEAR 1997}

\subsection{INTRODUCTION}

Fluor Daniel Hanford, Incorporated (FDH) and Bechtel Hanford, Incorporated (BHI) are responsible for monitoring radioactive and nonradioactive material released into the environment from U.S. Department of Energy (DOE) facilities and activities managed by them, on the Hanford Site.

This report fulfills the annual environmental release reporting requirements of DOE Order 5400.1. This report provides supplemental information to the Hanford Site Environmental Report for Calendar Year 1997 (PNNL-11795). The Hanford Site Environmental Report provides an update on the environmental status of the entire Hanford Site. The sitewide annual report summarizes the degree of compliance with applicable environmental regulations and informs the public concerning the impact of Hanford Site operations on the surrounding environment.

Like the Hanford Site Environmental Report, this annual report presents a summary of the environmental releases from facilities and activities. In addition to the summary data, this report also includes detailed data on air emissions, liquid effluents, and hazardous substances released to the environment from these facilities during calendar year 1997.

\subsection{TYPES AND LOCATIONS OF RELEASES}

Radioactive liquid effluents and air emissions are released from facilities in the 100,200 , 300,400 and 600 Areas. Radioactive liquid effluents are discharged to the soil in the 200 and 600 Areas, and to the Columbia River at the $100 \mathrm{~N}$ and $100 \mathrm{~K}$ Areas.

The major potential sources of nonradioactive air emissions of industrial origin are (1) fossil-fuel combustion emissions from the operation of powerhouses, package boilers, and portable generators, (2) emissions of nitrogen oxides, ammonia, and volatile organic compounds from liquid radioactive waste tanks, 242-A Evaporator, 200 Area Effluent Treatment Facility, and (3) carbon tetrachloride emissions from the $\mathrm{CCl}_{4}$ Vapor Extraction Project. The majority of these sources are located in the 200 and 300 Areas. In March 1997, the Department of Energy issued an Energy Savings Performance Contract (ESPC) to replace the Hanford Site's coal and oil fired boilers with smaller, cleaner operating, and more energy efficient diesel and natural gas fired boilers. In December 1997, operation of the 284-E and 284-W powerhouses ceased and 14 new diesel fired boilers came on line in the 200 Areas. In March 1998, operation of the 300 Area powerhouse ceased. 
Waste water from water treatment facilities and powerhouses located in the $100 \mathrm{~N}$ and 200 Areas is discharged to the soil column. In the 300 Areas waste water is sent to the 300 Area TEDF for treatment and discharged to the Columbia River, via a permitted outfall.

The $100 \mathrm{~N}$ Sanitary Sewage Lagoon receives sanitary waste water from the $100 \mathrm{~N}$ facilities and from failed septic systems, via tanker truck. $100 \mathrm{~B}, 100 \mathrm{D}, 100 \mathrm{H}$, and $100 \mathrm{~K}$ Areas discharge sanitary waste water into septic-tanks or drain-fields. Sanitary waste water is discharged to several septic-tank or subsurface disposal systems in the 200 Areas. Historically, sanitary waste water from the 300 and 400 Areas was discharged to a septic-tank trench system in the 300 Area and the sewage treatment plant and lagoon in the 400 Area. Sanitary waste water from the 300 Area is presently discharged to the city of Richland's publicly owned treatment works (POTW). In April of 1997, 400 Area sanitary waste water discharges started going to the Washington Public Power Supply's sewage treatment plant.

On March 29, 1996, the Solid Waste Landfill was closed. Leachate from the closed Solid Waste Landfill is collected, transported, and treated at the 300 Area TEDF. Since December 29, 1995, nonradioactive nonhazardous waste has been disposed at the city of Richland Landfill, which is adjacent to the southern edge of the Hanford Site boundary. Since February 1996, medical waste has been shipped to Waste Management of Kennewick for landfill disposal; asbestos has been shipped to Basin Disposal, Inc., in Pasco, and the Environmental Restoration Disposal Facility (ERDF), located on the Hanford Site, for landfill disposal. Since March of 1996, nonregulated containerized waste has been shipped to Waste Management of Kennewick.

\subsection{ENVIRONMENTAL RELEASE LIMITS AND GUIDELINES}

This section presents environmental release standards for radiological constituents. Relevant standards for nonradioactive constituents also are included in this section. Guidelines are applicable for constituents when the constituents: (1) affect the release and transport of radioactive constituents, (2) are necessary to meet any issued federal, state, or local permit, or (3) are necessary to meet any federal, state, or local regulations or guidelines prescribed by the U.S. Department of Energy, Richland Operations Office (DOE-RL).

\subsubsection{Limits for Radioactive Releases}

Quantities of radionuclides in air emissions and liquid effluents from Hanford Site facilities are governed by DOE Order 5400.5, Radiation Protection of the Public and the Environment. Quantities of radionuclides in air emissions are regulated by Title 40 of the Code of Federal Regulations (CFR) Part 61, Subpart $\mathrm{H}$ and the Washington Administrative Code (WAC) Chapter 246-247. The effective dose equivalent (EDE) received by any member of the offsite public from all effluents and emissions released during routine operations on the Hanford Site is not to exceed $100 \mathrm{mrem} / \mathrm{yr}(1 \mathrm{mSv} / \mathrm{yr})$ from continuous exposure throughout a prolonged 
period ( 5 years) and $500 \mathrm{mrem} / \mathrm{yr}(5 \mathrm{mSv} / \mathrm{yr})$ from noncontinuous, occasional exposure. From the air pathway only, the EDE to any member of the public is not to exceed $10 \mathrm{mrem} / \mathrm{yr}$ $(0.1 \mathrm{mSv} / \mathrm{yr})$.

The derived concentration guide (DCG) values in DOE Order 5400.5 apply at the location of actual exposure to members of the public. DCG values are not limits; these values are used for comparison purposes only.

The 300 Area TEDF is also regulated by an aquatic lands sewer outfall lease, Lease Number 20-012257, from the U.S. Department of Natural Resources. Limits for radioactive constituents include: $15 \mathrm{pCi} / \mathrm{L}\left(5.5 \mathrm{E}-04 \mathrm{~Bq} / \mathrm{m}^{3}\right)$ alpha, $50 \mathrm{pCi} / \mathrm{L}\left(1.9 \mathrm{E}+03 \mathrm{~Bq} / \mathrm{m}^{3}\right)$ beta, and $20,000 \mathrm{pCi} / \mathrm{L}\left(7.4 \mathrm{E}+05 \mathrm{~Bq} / \mathrm{m}^{3}\right)$ tritium.

Pacific Northwest National Laboratory (PNNL) issues the annual environmental summary report for the Hanford Site (PNNL-11795) as required by DOE Order 5400.1. This report assesses the radiological impact to the public resulting from all Hanford Site operations, in accordance with DOE Order 5400.5 and DOE Order 5480.1B. The PNNL report uses the release data contained in this report and the Radionuclide Air Emissions Report for the Hanford Site Calendar Year 1997 (DOE/RL-98-33) to calculate the offsite radiological dose impact. The PNNL report summarizes the information used to verify compliance with the dose standards specified in DOE Order 5400.5.

\subsubsection{Limits for Nonradioactive Releases}

The Clean Water Act of 1977, Comprehensive Environmental Response, Compensation, and Liability Act (CERCLA) of 1980, Resource Conservation and Recovery Act (RCRA) of 1976, Safe Drinking Water Act of 1974, Toxic Substances Control Act of 1976, and the State of Washington's regulations WAC 173-216, WAC 173-218, WAC 173-272, and WAC 173-303 also regulate nonradioactive constituents in air emissions and/or liquid effluents.

Liquid effluent streams discharging to the Columbia River are regulated by the National Pollutant Discharge Elimination System. Limits for specific constituents are specified in the permit issued by the U.S. Environmental Protection Agency (EPA).

Liquid effluent discharges to the soil column are permitted by the State of Washington Departments of Ecology (Ecology) and Health (WDOH), with the exception of storm water discharges. Limits for specific constituents are specified for each of the discharge permits issued by Ecology and WDOH. A permit application for storm water discharges to the soil column has been submitted, but a permit has not been issued. 
HNF-EP-0527-7

This page intentionally left blank. 


\subsection{AIR EMISSIONS}

Both radioactive and nonradioactive air emissions have been released to the atmosphere from facilities and activities managed by FDH and BHI. Release data for each type of emission are discussed separately.

\subsection{RADIONUCLIDE AIR EMISSIONS}

Radionuclide air emissions from actively ventilated point sources, with a potential to emit radioactive material to the atmosphere, are routinely monitored. Air emissions from actively ventilated point sources are usually discharged from stacks or vents. In the 200 Areas, stacks and vents are designated by a number that has a "291" or " 296 " prefix, depending on height: $61 \mathrm{~m}(200 \mathrm{ft})$ tall are designated by a "291" prefix; all other stacks and vents are designated by the "296" prefix. In the 100,300 , and 400 Areas, stacks and vents usually are identified by facility designations.

Radionuclide air emissions from sources other than actively ventilated point sources are monitored as diffuse and fugitive emissions. These sources are monitored collectively by the Near-Facility Monitoring Program and the Environmental Surveillance Program. Monitoring data from these sources is not presented in this report but can be obtained from the Radionuclide Air Emission Report for the Hanford Site Calendar Year 1997 (DOE/RL-98-33), the Hanford Site Near-Facility Environmental Monitoring Annual Report Calendar Year 1997 (HNF-EP-0573-6), the Hanford Site Environmental Monitoring Report for Calendar Year 1997 (PNNL-11795), and 1997 Surface Environmental Surveillance Data (PNNL-11796).

\subsubsection{Mitigation of Radionuclide Air Emissions}

The following are examples of methods used to remove radionuclides from air emissions: (1) high-efficiency particulate air (HEPA) filters, (2) sand filters, (3) charcoal absorbers (for iodine removal), (4) water scrubbers, (5) deep-bed fiberglass filters, and (6) fiberglass prefilters. Generally at least one stage, and often several stages, of HEPA filtration is used as the final particulate removal method before air is discharged to the atmosphere. All in-place HEPA filters are required to have an efficiency of $99.95 \%$ in removing airborne particles with a median aerodynamic equivalent diameter of $0.3 \mu \mathrm{m}$. Filter efficiency is routinely tested. Past release data have shown that radionuclide concentrations in many emissions are below the lower limit of analytical detection. 


\subsubsection{Radionuclide Air Emissions Data}

Release data on radionuclide air emissions from facilities, by area, are presented in Table 2-1. Tables 2-2 and 2-3 present data on the radionuclide air emissions from individual stacks and vents. The data consist of radionuclides detected or sampled for, average concentrations, and total activities.

Actively ventilated point source emissions are reported in this document when the following criteria were met during 1997: (1) point source requires continuous monitoring or periodic confirmatory measurements by 40 CFR 61, Subpart H, or WAC $246-247$, (2) point source is registered with WDOH, and (3) the point source normally has radionuclide emissions or potentially had radionuclide emissions. Point sources not included in this section did not meet the previous criteria or their air emissions were not forcibly discharged (e.g., passively ventilated, sealed off, deactivated). Air emissions forcibly discharged (actively ventilated) by exhaust fans are sampled only if radioactive material could potentially be released.

\subsection{NONRADIOACTIVE AIR EMISSIONS}

In 1997, the nonradioactive air emissions were discharged from the following facilities: 284-E powerhouse, 284-WB oil fired package boiler, 300 Area powerhouse, East Tank Farms, 242-A Evaporator, West Tank Farms, and 200 West Area $\mathrm{CCl}_{4}$ Vapor Extraction Project. Data on emissions from these sources are shown in Table 2-4. Powerhouse stack emissions were based on the quantity and type of fuel consumed, using formulas established by the EPA (EPA 450/4-90-003). Table 2-5 contains a summary of fuel consumption by the powerhouses.

Fabric-filter collection systems, called baghouses, remove particulate matter emitted from 284-E powerhouse. The $284-\mathrm{W}$ powerhouse's baghouses have been shutdown since February 1995. The 300 Area powerhouse has no emissions control system, since its boilers are oil fired. 


\section{Table 2-1}

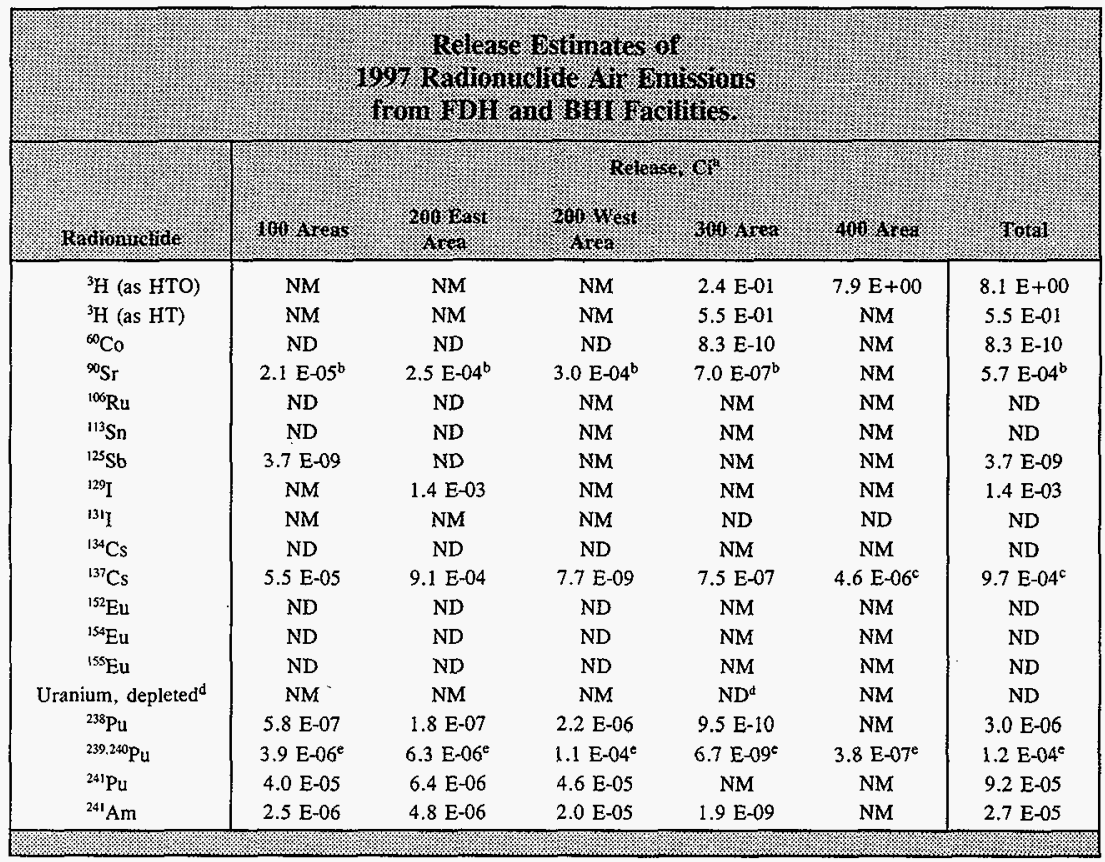

Notes:

a 1 curie $=3.7 \mathrm{E}+10$ becquerel; ND $=$ not detected (i.e., either the radionuclide was not detected in any sample during the year, or the average of all the measurements for that given radionuclide or type of radioactivity made during the year was below background levels): $\mathrm{NM}=$ not measured.

b This value includes total beta release data. Total beta results assumed to be ${ }^{90} \mathrm{~S} r$ for dose calculations.

c This value includes total beta release data. Total beta results assumed to be ${ }^{137} \mathrm{Cs}$ for dose calculations from FFTF emissions.

d Determined from total alpha measurements. Assumed to be depleted uranium consisting of $63.478 \mathrm{Ci} \%{ }^{238} \mathrm{U}, 0.821 \mathrm{Ci} \%$ ${ }^{235} \mathrm{U}$, and $35.701 \mathrm{Ci} \%{ }^{234} \mathrm{U}\left(99.797 \mathrm{Wt} \%{ }^{238} \mathrm{U}, 0.200 \mathrm{Wt} \%{ }^{235} \mathrm{U}\right.$, and $\left.0.003 \mathrm{Wt} \%{ }^{234} \mathrm{U}\right)$.

e This value includes total alpha release data. Total alpha results assumed to be ${ }^{239 / 240} \mathrm{Pu}$ for dose calculations. 
Table 2-2

(4 sheets)

\begin{tabular}{|c|c|c|c|c|c|c|}
\hline 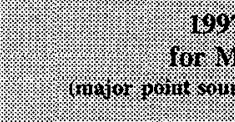 & \%ongon & 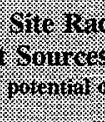 & buinging & 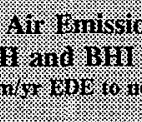 & 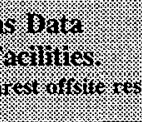 & \%x. \\
\hline 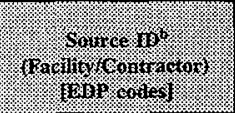 & 3isis & 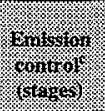 & 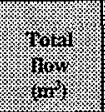 & 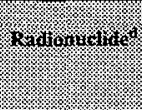 & 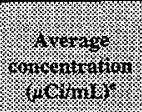 & 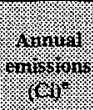 \\
\hline & & 200 East & rea Point S & Irces & & \\
\hline $\begin{array}{l}\text { 291-A-1 } \\
\text { (PUREX/FDH) } \\
\text { [A552,A511,A007] }\end{array}$ & 61.0 & HEPA (3) & $7.9 \mathrm{E}+08$ & $\begin{array}{l}{ }^{90} \mathrm{Sr} \\
{ }^{106} \mathrm{Ru} \\
{ }^{113} \mathrm{Sn} \\
{ }^{125} \mathrm{Sb} \\
{ }^{129} \mathrm{I} \\
{ }^{134} \mathrm{Cs} \\
{ }^{137} \mathrm{Cs} \\
{ }^{238} \mathrm{Pu} \\
{ }^{239.240} \mathrm{Pu} \\
{ }^{241} \mathrm{Pu} \\
{ }^{241} \mathrm{Am} \\
\text { total alpha } \\
\text { total beta }\end{array}$ & $\begin{array}{c}1.9 \mathrm{E}-14 \\
\mathrm{ND} \\
\mathrm{ND} \\
\mathrm{ND} \\
1.5 \mathrm{E}-12 \\
\mathrm{ND} \\
4.2 \mathrm{E}-14 \\
1.9 \mathrm{E}-16 \\
3.8 \mathrm{E}-15 \\
8.1 \mathrm{E}-15 \\
5.2 \mathrm{E}-15 \\
9.2 \mathrm{E}-15 \\
6.0 \mathrm{E}-14\end{array}$ & $\begin{array}{c}1.5 \mathrm{E}-05 \\
\mathrm{ND} \\
\mathrm{ND} \\
\mathrm{ND} \\
1.2 \mathrm{E}-03 \\
\mathrm{ND} \\
3.1 \mathrm{E}-05 \\
1.5 \mathrm{E}-07 \\
3.1 \mathrm{E}-06 \\
6.4 \mathrm{E}-06 \\
4.2 \mathrm{E}-06 \\
7.4 \mathrm{E}-06 \\
4.7 \mathrm{E}-05\end{array}$ \\
\hline $\begin{array}{l}291-\mathrm{B}-1 \\
\text { (B Plant/FDH) } \\
{[\mathrm{B} 691]}\end{array}$ & 61.0 & HEPA (2) & $6.1 \mathrm{E}+08$ & $\begin{array}{l}{ }^{90} \mathrm{Sr} \\
{ }^{134} \mathrm{Cs} \\
{ }^{137} \mathrm{Cs} \\
{ }^{238} \mathrm{Pu} \\
{ }^{239.240} \mathrm{Pu} \\
{ }^{241} \mathrm{Am} \\
\text { total alpha } \\
\text { total beta }\end{array}$ & $\begin{array}{l}6.3 \text { E-14 } \\
\text { ND } \\
1.3 \text { E-12 } \\
4.2 \text { E-17 } \\
4.7 \text { E-15 } \\
9.4 \text { E-16 } \\
2.7 \text { E-15 } \\
8.5 \text { E-13 }\end{array}$ & $\begin{array}{c}3.9 \mathrm{E}-05 \\
\text { ND } \\
7.9 \mathrm{E}-04 \\
2.6 \mathrm{E}-08 \\
2.9 \mathrm{E}-06 \\
5.7 \mathrm{E}-07 \\
1.7 \mathrm{E}-06 \\
5.1 \mathrm{E}-04\end{array}$ \\
\hline $\begin{array}{l}296-\mathrm{A}-22 \\
\text { (242-A Evaporator/FDH) } \\
{[\mathrm{E} 643, \mathrm{E} 002]}\end{array}$ & 18.6 & HEPA (2) & $6.3 E+06$ & $\begin{array}{l}{ }^{90} \mathrm{Sr} \\
{ }^{106} \mathrm{Ru} \\
{ }^{113} \mathrm{Sn} \\
{ }^{125} \mathrm{Sb} \\
{ }^{129} \mathrm{I} \\
{ }^{134} \mathrm{Cs} \\
{ }^{137} \mathrm{Cs} \\
{ }^{238} \mathrm{Pu} \\
{ }^{239.240} \mathrm{Pu} \\
{ }^{241} \mathrm{Am} \\
\text { total alpha } \\
\text { total beta }\end{array}$ & $\begin{array}{l}5.2 \mathrm{E}-16 \\
\text { ND } \\
\text { ND } \\
\text { ND } \\
\text { ND } \\
\text { ND } \\
\text { ND } \\
\text { ND } \\
1.3 \mathrm{E}-17 \\
6.6 \mathrm{E}-17 \\
9.2 \mathrm{E}-16 \\
3.7 \mathrm{E}-15\end{array}$ & $\begin{array}{c}3.3 \mathrm{E}-09 \\
\text { ND } \\
\text { ND } \\
\text { ND } \\
\text { ND } \\
\text { ND } \\
\text { ND } \\
\text { ND } \\
\text { 8.1 E-11 } \\
4.1 \text { E-10 } \\
5.8 \text { E-10 } \\
2.3 \text { E-08 }\end{array}$ \\
\hline $\begin{array}{l}296-A-12 \\
\text { (East Tank Farms/FDH) } \\
{[\text { E058] }}\end{array}$ & 45.7 & HEPA (2) & $0.0 \mathrm{E}+00$ & - ${ }^{0} \mathrm{Sr}$ & (did not & rate) \\
\hline
\end{tabular}


Table 2-2

(4 sheets)

\begin{tabular}{|c|c|c|c|c|c|c|}
\hline . & (2) & mitore & grimalu & 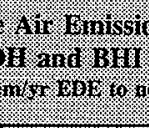 & 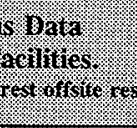 & $n^{2}$ \\
\hline 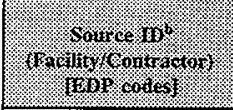 & 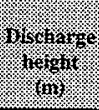 & 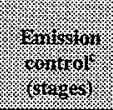 & 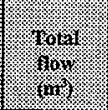 & 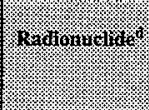 & 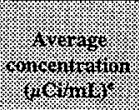 & 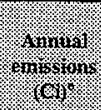 \\
\hline \begin{tabular}{|l}
$296-\mathrm{A}-17$ \\
296-P-26 (backup) \\
(East Tank Farms/FDH) \\
[E059.E026,E027] \\
[E039,E040,E041]
\end{tabular} & 15.2 & HEPA (2) & $4.8 \mathrm{E}+07$ & $\begin{array}{l}{ }^{90} \mathrm{Sr} \\
{ }^{106} \mathrm{Ru} \\
{ }^{113} \mathrm{Sn} \\
{ }^{125} \mathrm{Sb} \\
{ }^{129} \mathrm{I} \\
{ }^{134} \mathrm{Cs} \\
{ }^{137} \mathrm{Cs} \\
{ }^{238} \mathrm{Pu} \\
{ }^{239.200} \mathrm{Pu} \\
{ }^{24} \mathrm{Am} \\
\text { total alpha } \\
\text { total beta }\end{array}$ & $\begin{array}{c}3.5 \mathrm{E}-14 \\
\mathrm{ND} \\
\mathrm{ND} \\
\mathrm{ND} \\
4.6 \mathrm{E}-12 \\
\mathrm{ND} \\
4.6 \mathrm{E}-13 \\
2.0 \mathrm{E}-17 \\
1.4 \mathrm{E}-16 \\
1.4 \mathrm{E}-16 \\
6.6 \mathrm{E}-16 \\
3.7 \mathrm{E}-13\end{array}$ & $\begin{array}{c}1.6 \mathrm{E}-06 \\
\text { ND } \\
\text { ND } \\
\text { ND } \\
2.1 \mathrm{E}-04 \\
\text { ND } \\
2.0 \mathrm{E}-05 \\
8.8 \mathrm{E}-10 \\
6.9 \mathrm{E}-09 \\
7.5 \mathrm{E}-09 \\
2.9 \mathrm{E}-08 \\
1.7 \mathrm{E}-05\end{array}$ \\
\hline $\begin{array}{l}296-\mathrm{A}-25 \\
\text { (East Tank Farms/FDH) } \\
\text { [E080] }\end{array}$ & 3.0 & HEPA (2) & $1.1 \mathrm{E}+06$ & $\begin{array}{l}{ }^{90} \mathrm{Sr} \\
{ }^{134} \mathrm{Cs} \\
{ }^{137} \mathrm{Cs} \\
{ }^{238} \mathrm{Pu} \\
{ }^{239.240} \mathrm{Pu} \\
{ }^{241} \mathrm{Am} \\
\text { total alpha } \\
\text { total beta }\end{array}$ & $\begin{array}{c}\text { ND } \\
\text { ND } \\
5.8 \text { E-13 } \\
\text { ND } \\
5.2 \text { E-17 } \\
1.2 \text { E-16 } \\
8.0 \text { E-16 } \\
5.7 \text { E-13 }\end{array}$ & $\begin{array}{c}\text { ND } \\
\text { ND } \\
6.4 \mathrm{E}-07 \\
5.7 \mathrm{E}-11 \\
\text { ND } \\
1.4 \mathrm{E}-10 \\
8.8 \mathrm{E}-10 \\
6.3 \mathrm{E}-07\end{array}$ \\
\hline $\begin{array}{l}\text { 296-B-28 } \\
\text { (West Tank Farms/FDH) } \\
\text { [E886] }\end{array}$ & 3.4 & HEPA (2) & $3.1 E+06$ & $\begin{array}{l}{ }^{90} \mathrm{Sr} \\
{ }^{134} \mathrm{Cs} \\
{ }^{137} \mathrm{Cs} \\
{ }^{238} \mathrm{Pu} \\
{ }^{239.240} \mathrm{Pu} \\
\text { - }{ }^{241} \mathrm{Am} \\
\text { total alpha } \\
\text { total beta }\end{array}$ & $\begin{array}{c}5.6 \text { E-16 } \\
\text { ND } \\
2.5 \text { E-16 } \\
\text { ND } \\
4.2 \text { E-17 } \\
1.2 \text { E-16 } \\
3.8 \text { E-16 } \\
5.4 \text { E-15 }\end{array}$ & $\begin{array}{c}1.7 \mathrm{E}-09 \\
\text { ND } \\
7.4 \mathrm{E}-10 \\
\text { ND } \\
1.3 \mathrm{E}-10 \\
3.6 \mathrm{E}-10 \\
1.1 \mathrm{E}-09 \\
1.7 \mathrm{E}-08\end{array}$ \\
\hline $\begin{array}{l}296-\mathrm{C}-5 \\
\text { (East Tank Farms/FDH) } \\
{[\text { E069] }}\end{array}$ & 14.6 & HEPA (2) & $4.8 \mathrm{E}+07$ & $\begin{array}{l}\cdot{ }^{\circ 9} \mathrm{Sr} \\
{ }^{136} \mathrm{Cs} \\
{ }^{137} \mathrm{Cs} \\
{ }^{238} \mathrm{Pu} \\
{ }^{239.240} \mathrm{Pu} \\
\cdot{ }^{241} \mathrm{Am} \\
\text { total alpha } \\
\text { total beta }\end{array}$ & $\begin{array}{c}1.9 \mathrm{E}-15 \\
\text { ND } \\
3.5 \mathrm{E}-15 \\
\mathrm{ND} \\
2.4 \mathrm{E}-17 \\
4.7 \mathrm{E}-17 \\
1.7 \mathrm{E}-16 \\
8.9 \mathrm{E}-15\end{array}$ & $\begin{array}{c}9.2 \mathrm{E}-08 \\
\text { ND } \\
1.7 \mathrm{E}-07 \\
\text { ND } \\
1.2 \mathrm{E}-09 \\
2.2 \mathrm{E}-09 \\
8.1 \mathrm{E}-09 \\
4.3 \mathrm{E}-07\end{array}$ \\
\hline $\begin{array}{l}\text { 296-P-16 } \\
\text { (East Tank Farms/FDH) } \\
\text { [E068] }\end{array}$ & 4.6 & HEPA (2) & $4.9 \mathrm{E}+07$ & $\begin{array}{l}{ }^{90} \mathrm{Sr} \\
{ }^{134} \mathrm{Cs} \\
{ }^{137} \mathrm{Cs} \\
{ }^{238} \mathrm{Pu} \\
{ }^{239,240} \mathrm{Pu} \\
{ }^{241} \mathrm{Am} \\
\text { total alpha } \\
\text { total beta }\end{array}$ & $\begin{array}{l}3.8 \text { E-15 } \\
\text { ND } \\
1.1 \text { E-14 } \\
4.0 \text { E-18 } \\
9.5 \text { E-17 } \\
1.1 \text { E-16 } \\
6.2 \text { E-17 } \\
1.4 \text { E-14 }\end{array}$ & $\begin{array}{c}1.7 \mathrm{E}-07 \\
\text { ND } \\
4.8 \mathrm{E}-07 \\
1.8 \mathrm{E}-10 \\
4.3 \mathrm{E}-09 \\
4.8 \mathrm{E}-09 \\
2.8 \mathrm{E}-09 \\
6.2 \mathrm{E}-07\end{array}$ \\
\hline
\end{tabular}


Table 2-2

(4 sheets)

\begin{tabular}{|c|c|c|c|c|c|c|}
\hline \multicolumn{7}{|c|}{ 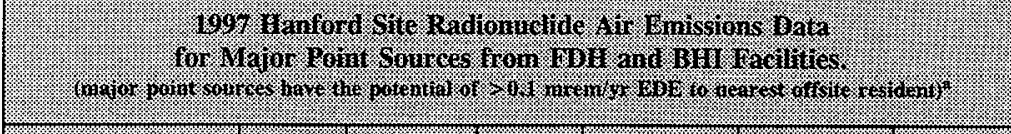 } \\
\hline (2. & 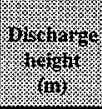 & 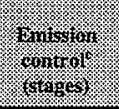 & (3) & 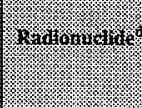 & 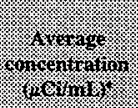 & 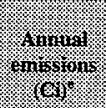 \\
\hline $\begin{array}{l}\text { 296-P-32 } \\
\text { (East Tank Farms/FDH) } \\
\text { [E401] }\end{array}$ & 4.6 & HEPA (2) & $1.4 \mathrm{E}+03$ & $\begin{array}{l}\text { - } \\
\text { total alpha } \\
\text { total beta }\end{array}$ & $\begin{array}{l}\mathrm{NM} \\
\mathrm{ND} \\
1.5 \mathrm{E}-12\end{array}$ & $\begin{array}{l}\text { NM } \\
\text { ND } \\
2.1 \mathrm{E}-09\end{array}$ \\
\hline \multicolumn{7}{|c|}{200 West Area Point Sources } \\
\hline \begin{tabular}{|l}
$291-\mathrm{Z}-1$ \\
(PFP/FDH) \\
{$[\mathrm{Z} 810]$}
\end{tabular} & 61.0 & HEPA (1-3) & $4.3 \mathrm{E}+09$ & $\begin{array}{l}{ }^{238} \mathrm{Pu} \\
{ }^{239,240} \mathrm{Pu} \\
{ }^{241} \mathrm{Pu} \\
{ }^{241} \mathrm{Am} \\
\text { total alpha } \\
\text { total beta }\end{array}$ & $\begin{array}{ll}5.0 & \mathrm{E}-16 \\
2.1 & \mathrm{E}-14 \\
1.1 & \mathrm{E}-14 \\
4.7 & \mathrm{E}-15 \\
2.4 & \mathrm{E}-14 \\
2.8 & \mathrm{E}-15\end{array}$ & $\begin{array}{ll}2.2 & \mathrm{E}-06 \\
9.3 & \mathrm{E}-05 \\
4.6 & \mathrm{E}-05 \\
2.0 & \mathrm{E}-05 \\
1.1 & \mathrm{E}-04 \\
1.2 & \mathrm{E}-05\end{array}$ \\
\hline $\begin{array}{l}\text { 296-S-22 } \\
\text { (West Tank Farms/FDH) } \\
\text { [W880] }\end{array}$ & 3.7 & HEPA (2) & $2.1 \mathrm{E}+06$ & $\begin{array}{l}{ }^{10} \mathrm{Sr} \\
{ }^{134} \mathrm{Cs} \\
{ }^{137} \mathrm{Cs} \\
{ }^{238} \mathrm{Pu} \\
{ }^{239.240} \mathrm{Pu} \\
\text { - }{ }^{241} \mathrm{Am} \\
\text { total alpha } \\
\text { total beta }\end{array}$ & $\begin{array}{c}3.2 \mathrm{E}-15 \\
\text { ND } \\
2.4 \mathrm{E}-16 \\
\text { ND } \\
2.2 \mathrm{E}-17 \\
5.3 \mathrm{E}-17 \\
2.5 \mathrm{E}-16 \\
4.6 \mathrm{E}-15\end{array}$ & $\begin{array}{c}6.6 \mathrm{E}-09 \\
\text { ND } \\
5.0 \mathrm{E}-10 \\
\text { ND } \\
4.7 \text { E-11 } \\
1.1 \text { E-10 } \\
5.2 \text { E-10 } \\
9.5 \text { E-09 }\end{array}$ \\
\hline $\begin{array}{l}\text { 296-T-18 } \\
\text { (West Tank Farms/FDH) } \\
\text { [W882] }\end{array}$ & 3.7 & HEPA (2) & $3.5 \mathrm{E}+06$ & $\begin{array}{l}{ }^{90} \mathrm{Sr} \\
{ }^{134} \mathrm{Cs} \\
{ }^{137} \mathrm{Cs} \\
{ }^{238} \mathrm{Pu} \\
\cdot{ }^{239.240} \mathrm{Pu} \\
{ }^{241} \mathrm{Am} \\
\text { total alpha } \\
\text { total beta }\end{array}$ & $\begin{array}{c}\text { ND } \\
\text { ND } \\
2.0 \text { E-15 } \\
2.4 \text { E-18 } \\
7.4 \text { E-17 } \\
1.4 \text { E-16 } \\
5.0 \text { E-16 } \\
4.9 \text { E-15 }\end{array}$ & $\begin{array}{c}\text { ND } \\
\text { ND } \\
7.2 \mathrm{E}-09 \\
8.6 \mathrm{E}-12 \\
2.6 \mathrm{E}-10 \\
5.1 \mathrm{E}-10 \\
1.8 \mathrm{E}-09 \\
1.7 \mathrm{E}-08\end{array}$ \\
\hline \multicolumn{7}{|c|}{300 Area Point Sources } \\
\hline $\begin{array}{l}\text { 340-NT-EX } \\
\text { (340 Waste } \\
\text { Handling/FDH) } \\
\text { [F002,F007] }\end{array}$ & 5.5 & HEPA (2) & $2.6 \mathrm{E}+07$ & $\begin{array}{l}{ }^{131} \mathrm{I} \\
{ }^{137} \mathrm{Cs} \\
{ }^{238} \mathrm{Pu} \\
{ }^{239.240} \mathrm{Pu} \\
{ }^{241} \mathrm{Am} \\
\text { total alpha } \\
\text { total beta }\end{array}$ & $\begin{array}{c}\text { ND } \\
\text { ND } \\
\text { ND } \\
\text { ND } \\
\text { ND } \\
4.5 \mathrm{E}-17 \\
2.6 \mathrm{E}-16\end{array}$ & $\begin{array}{c}\text { ND } \\
\text { ND } \\
\text { ND } \\
\text { ND } \\
\text { ND } \\
1.2 \mathrm{E}-09 \\
6.9 \mathrm{E}-09\end{array}$ \\
\hline
\end{tabular}


HNF-EP-0527-7

Table 2-2

(4 sheets)

\begin{tabular}{|c|c|c|c|c|c|c|}
\hline 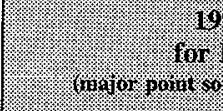 & 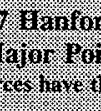 & 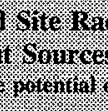 & Wome & 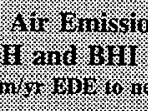 & 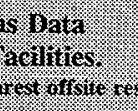 & (1) \\
\hline 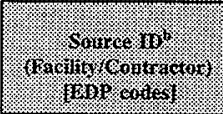 & 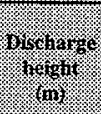 & Hongon & 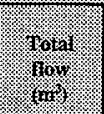 & 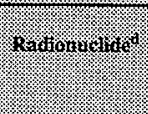 & 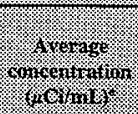 & 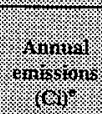 \\
\hline $\begin{array}{l}\text { EP-324-01-S } \\
\text { (324 Bldg./FDH) } \\
{[\mathrm{F} 025, \mathrm{~F} 028]}\end{array}$ & 48.0 & HEPA (2) & $1.0 \mathrm{E}+09$ & $\begin{array}{l}{ }^{3} \mathrm{H} \text { (as HTO }{ }^{f} \\
{ }^{3} \mathrm{H} \text { (as HT) } \\
{ }^{90} \mathrm{Sr} \\
{ }^{137} \mathrm{Cs} \\
{ }^{238} \mathrm{Pu} \\
{ }^{239} \cdot 240 \mathrm{Pu} \\
{ }^{241} \mathrm{Am} \\
\text { total alpha } \\
\text { total beta }\end{array}$ & $\begin{array}{c}1.3 \mathrm{E}-10 \\
4.8 \mathrm{E}-10 \\
3.8 \mathrm{E}-17 \\
\mathrm{ND} \\
\mathrm{ND} \\
\mathrm{ND} \\
\mathrm{ND} \\
\mathrm{ND} \\
1.5 \mathrm{E}-16\end{array}$ & $\begin{array}{l}1.3 \mathrm{E}-01 \\
4.9 \mathrm{E}-01 \\
3.9 \mathrm{E}-08 \\
\mathrm{ND} \\
\mathrm{ND} \\
\mathrm{ND} \\
\mathrm{ND} \\
\mathrm{ND} \\
1.6 \mathrm{E}-07\end{array}$ \\
\hline $\begin{array}{l}\text { EP-327-01-S } \\
\text { (327 Bldg./FDH) } \\
{[\text { F026,F029] }}\end{array}$ & 27.1 & HEPA (2) & $1.5 \mathrm{E}+09$ & $\begin{array}{l}{ }^{3} \mathrm{H} \text { (as HTO) } \\
{ }^{3} \mathrm{H} \text { (as HT) } \\
{ }^{90} \mathrm{Sr} \\
{ }^{137} \mathrm{Cs} \\
{ }^{220} \mathrm{Rn} \\
{ }^{222} \mathrm{Rn} \\
{ }^{238} \mathrm{Pu} \\
{ }^{239.240} \mathrm{Pu} \\
{ }^{241} \mathrm{Am} \\
\text { total alpha } \\
\text { total beta }\end{array}$ & 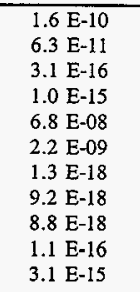 & $\begin{array}{c}1.1 \mathrm{E}-01 \\
4.6 \mathrm{E}-02 \\
2.3 \mathrm{E}-07 \\
7.5 \mathrm{E}-07 \\
5.0 \mathrm{E}+01 \\
1.6 \mathrm{E}+00 \\
9.5 \mathrm{E}-10 \\
6.7 \mathrm{E}-09 \\
6.4 \mathrm{E}-09 \\
8.1 \mathrm{E}-08 \\
2.3 \mathrm{E}-06\end{array}$ \\
\hline $\begin{array}{l}\text { EP-327-02-V } \\
\text { (327 Decon. Cell/FDH) } \\
\text { [F027] }\end{array}$ & 14.0 & HEPA (2) & $1.1 \mathrm{E}+07$ & $\begin{array}{l}{ }^{60} \mathrm{Co} \\
{ }^{90} \mathrm{Sr} \\
{ }^{137} \mathrm{Cs} \\
{ }^{238} \mathrm{Pu} \\
{ }^{239,240} \mathrm{Pu} \\
{ }^{241} \mathrm{Am} \\
\text { total alpha } \\
\text { total beta }\end{array}$ & $\begin{array}{l}7.5 \mathrm{E}-17 \\
\mathrm{ND} \\
2.1 \mathrm{E}-16 \\
\mathrm{ND} \\
\mathrm{ND} \\
1.0 \mathrm{E}-18 \\
\mathrm{ND} \\
5.6 \mathrm{E}-16\end{array}$ & $\begin{array}{l}8.3 \mathrm{E}-10 \\
\mathrm{ND} \\
2.3 \mathrm{E}-09 \\
\mathrm{ND} \\
\mathrm{ND} \\
1.1 \mathrm{E}-11 \\
\mathrm{ND} \\
2.3 \mathrm{E}-06\end{array}$ \\
\hline
\end{tabular}

Notes:

a Determining the state of National Emission Standards for Hazardous Air Pollutants Subpart H compliance for each point source involved using nearest offsite residences, which differed from the MEI; EDE = effective dose equivalent.

b ID = identification, i.e., the alpha-numeric designator for the respective point source; EDP Code = electronic data processing code for sampler identification; FDH = Fluor Daniel Hanford, Inc.; BHI = Bechtel Hanford, Inc.

c Efficiencies are: $\geq 99.95 \%$ for HEPA; $\geq 95 \%$ for charcoal; $\geq 99.8 \%$ for sand filter; $0 \%$ for no emission control; HEPA $=$ high efficiency particulate air filter.

d Bullets, " $\bullet "$, identify specific radionuclides sampling and analysis required by 40 CFR 61 Subpart H.

e $1 \mu \mathrm{Ci} / \mathrm{mL}=3.7 \mathrm{E}+10 \mathrm{~Bq} / \mathrm{m}^{3} ; 1$ curie $=3.7 \mathrm{E}+10$ becquerel; $\mathrm{ND}=$ not detected (i.e., either the radionuclide was not detected in any sample during the year, or the average of all the measurements for that given radionuclide or type of radioactivity made during the year was below background levels).

f $\mathrm{HTO}$ is tritium as condensable water vapor; HT is tritium as incondensable gas. 
Table 2-3

(7 sheets)

\begin{tabular}{|c|c|c|c|c|c|c|}
\hline 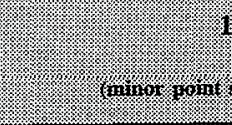 & 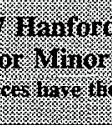 & Ming Rain & 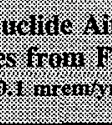 & 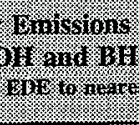 & 2lon & \\
\hline 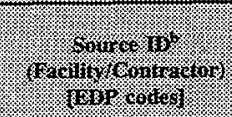 & 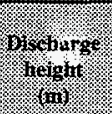 & 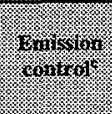 & 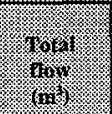 & han & 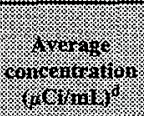 & 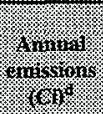 \\
\hline & & 100 Area & oint Sources & & & \\
\hline $\begin{array}{l}116-\mathrm{N} \\
(100 \mathrm{~N} \text { Area/BHI }) \\
{[\mathrm{Y} 211, \mathrm{Y} 212, \mathrm{Y} 213]}\end{array}$ & 61.3 & $\begin{array}{l}\text { HEPA, } \\
\text { chatcoal }\end{array}$ & $1.3 \mathrm{E}+09$ & $\begin{array}{l}{ }^{60} \mathrm{Co} \\
{ }^{90} \mathrm{Sr} \\
{ }^{137} \mathrm{Cs} \\
{ }^{233} \mathrm{Pu} \\
{ }^{239240} \mathrm{Pu} \\
{ }^{241} \mathrm{Am} \\
\text { total alpha } \\
\text { total beta }\end{array}$ & $\begin{array}{c}\text { ND } \\
2.2 \mathrm{E}-15 \\
\text { ND } \\
\text { ND } \\
2.1 \mathrm{E}-17 \\
1.8 \mathrm{E}-16 \\
1.0 \mathrm{E}-16 \\
4.5 \mathrm{E}-15\end{array}$ & $\begin{array}{c}\text { ND } \\
2.8 \mathrm{E}-06 \\
\text { ND } \\
\text { ND } \\
2.6 \mathrm{E}-08 \\
2.2 \mathrm{E}-07 \\
1.3 \mathrm{E}-07 \\
5.8 \mathrm{E}-06\end{array}$ \\
\hline $\begin{array}{l}107-\mathrm{N} \\
(100 \mathrm{~N} \text { Area/BHI }) \\
{[\mathrm{Y} 265, \mathrm{Y} 266]}\end{array}$ & 12.0 & HEPA & $1.1 \mathrm{E}+08$ & $\begin{array}{l}\text { total alpha } \\
\text { total beta }\end{array}$ & $\begin{array}{c}\text { ND } \\
1.2 \mathrm{E}-15\end{array}$ & $\begin{array}{c}\mathrm{ND} \\
1.8 \mathrm{E}-07\end{array}$ \\
\hline $\begin{array}{l}\text { RCF-1-EX } \\
(100 \mathrm{~N} \text { Area/BHI) } \\
{[\mathrm{Y} 215]}\end{array}$ & 3.0 & HEPA & $2.6 \mathrm{E}+05$ & $\begin{array}{l}\text { total alpha } \\
\text { total beta }\end{array}$ & $\begin{array}{l}1.8 \mathrm{E}-15 \\
3.2 \mathrm{E}-15\end{array}$ & $\begin{array}{l}4.6 \text { E-10 } \\
8.2 \text { E-10 }\end{array}$ \\
\hline $\begin{array}{l}\text { 105-KE Basin } \\
\text { (100 K Area/FDH) } \\
{[\mathrm{Y} 245-\mathrm{Y} 248]}\end{array}$ & 12.8 & none & $6.8 E+08$ & $\begin{array}{l}{ }^{60} \mathrm{Co} \\
{ }^{90} \mathrm{Sr} \\
{ }^{106} \mathrm{Ru} \\
{ }^{125} \mathrm{Sb} \\
{ }^{134} \mathrm{Cs} \\
{ }^{137} \mathrm{Cs} \\
{ }^{154} \mathrm{Eu} \\
{ }^{155} \mathrm{Eu} \\
{ }^{238} \mathrm{Pu} \\
{ }^{239 / 240} \mathrm{Pu} \\
{ }^{241} \mathrm{Pu} \\
{ }^{241} \mathrm{Am} \\
\text { total alpha } \\
\text { tota! beta }\end{array}$ & $\begin{array}{c}\text { ND } \\
2.3 \mathrm{E}-14 \\
\text { ND } \\
5.5 \mathrm{E}-18 \\
\text { ND } \\
6.9 \mathrm{E}-14 \\
\text { ND } \\
\text { ND } \\
8.4 \mathrm{E}-16 \\
5.3 \mathrm{E}-15 \\
5.7 \mathrm{E}-14 \\
3.3 \mathrm{E}-15 \\
1.4 \mathrm{E}-14 \\
1.3 \mathrm{E}-13\end{array}$ & $\begin{array}{c}\text { ND } \\
1.6 \mathrm{E}-05 \\
\text { ND } \\
3.7 \mathrm{E}-09 \\
\text { ND } \\
4.8 \mathrm{E}-05 \\
\text { ND } \\
\text { ND } \\
5.7 \mathrm{E}-07 \\
3.6 \mathrm{E}-06 \\
3.9 \mathrm{E}-05 \\
2.3 \mathrm{E}-06 \\
9.9 \mathrm{E}-06 \\
9.3 \mathrm{E}-05\end{array}$ \\
\hline
\end{tabular}


Table 2-3

(7 sheets)

\begin{tabular}{|c|c|c|c|c|c|c|}
\hline 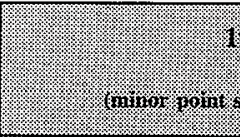 & 7\% Harom & 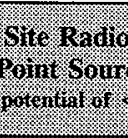 & 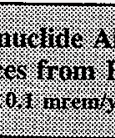 & 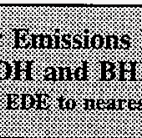 & Hat. & \\
\hline 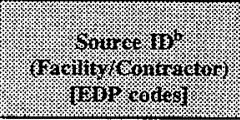 & 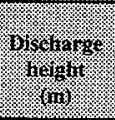 & 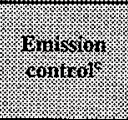 & $\operatorname{lng}^{2}$ & 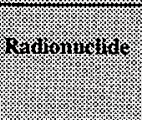 & 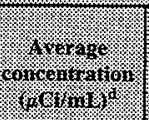 & 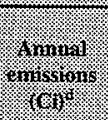 \\
\hline $\begin{array}{l}\text { 105-KW Basin } \\
\text { (100 K Area/FDH) } \\
\text { [Y234-Y236] }\end{array}$ & 12.8 & none & $4.2 \mathrm{E}+08$ & $\begin{array}{l}{ }^{60} \mathrm{Co} \\
{ }^{90} \mathrm{Sr} \\
{ }^{106} \mathrm{Ru} \\
{ }^{125} \mathrm{Sb} \\
{ }^{134} \mathrm{Cs} \\
{ }^{137} \mathrm{Cs} \\
{ }^{154} \mathrm{Eu} \\
{ }^{155} \mathrm{Eu} \\
{ }^{238} \mathrm{Pu} \\
{ }^{239} 92^{40} \mathrm{Pu} \\
{ }^{24 !} \mathrm{Pu} \\
{ }^{241} \mathrm{Am} \\
\text { total alpha } \\
\text { total beta }\end{array}$ & $\begin{array}{c}5.3 \mathrm{E}-18 \\
2.0 \mathrm{E}-15 \\
\mathrm{ND} \\
\mathrm{ND} \\
\mathrm{ND} \\
1.6 \mathrm{E}-14 \\
\mathrm{ND} \\
\mathrm{ND} \\
1.6 \mathrm{E}-17 \\
9.0 \mathrm{E}-17 \\
1.4 \mathrm{E}-15 \\
1.2 \mathrm{E}-16 \\
3.2 \mathrm{E}-15 \\
3.8 \mathrm{E}-14\end{array}$ & $\begin{array}{c}2.3 \mathrm{E}-09 \\
8.3 \mathrm{E}-07 \\
\mathrm{ND} \\
\mathrm{ND} \\
\mathrm{ND} \\
7.1 \mathrm{E}-06 \\
\mathrm{ND} \\
\mathrm{ND} \\
6.8 \mathrm{E}-09 \\
3.8 \mathrm{E}-08 \\
6.2 \mathrm{E}-07 \\
4.9 \mathrm{E}-08 \\
1.4 \mathrm{E}-06 \\
1.6 \mathrm{E}-05\end{array}$ \\
\hline $\begin{array}{l}\text { 1706-KER -27 ft } \\
(100 \mathrm{~K} \text { Area/FDH) } \\
{[\mathrm{Y} 244]}\end{array}$ & 0.9 & HEPA & $1.6 \mathrm{E}+06$ & $\begin{array}{l}\text { total alpha } \\
\text { total beta }\end{array}$ & $\begin{array}{l}\text { ND } \\
\text { ND }\end{array}$ & $\begin{array}{l}\text { ND } \\
\text { ND }\end{array}$ \\
\hline $\begin{array}{l}\text { 1706-KE } \\
(100 \mathrm{~K} \mathrm{Area/FDH}) \\
{[Y 243]}\end{array}$ & $\overline{7.6}$ & HEPA & $9.1 \mathrm{E}+07$ & $\begin{array}{l}\text { total alpha } \\
\text { total beta }\end{array}$ & $\begin{array}{l}2.2 \mathrm{E}-15 \\
1.2 \mathrm{E}-14\end{array}$ & $\begin{array}{l}1.9 \mathrm{E}-07 \\
1.1 \mathrm{E}-06\end{array}$ \\
\hline & & 200 East Are & Point Sourc & & & \\
\hline $\begin{array}{l}296-\mathrm{B}-5 \\
\text { (B Plant/FDH) } \\
\text { [B686]. }\end{array}$ & 3.7 & HEPA & $1.0 \mathrm{E}+07$ & $\begin{array}{l}\text { total alpha } \\
\text { total beta }\end{array}$ & $\begin{array}{l}9.7 \text { E-16 } \\
4.8 \text { E-15 }\end{array}$ & $\begin{array}{l}9.9 \mathrm{E}-09 \\
4.9 \mathrm{E}-08\end{array}$ \\
\hline $\begin{array}{l}296-\mathrm{B}-10 \\
\text { (WESF/FDH) } \\
{[\mathrm{B} 748]}\end{array}$ & 22.9 & HEPA & $3.1 \mathrm{E}+08$ & $\begin{array}{l}{ }^{90} \mathrm{Sr} \\
{ }^{134} \mathrm{Cs} \\
{ }^{133} \mathrm{Cs} \\
\text { total aipha } \\
\text { total beta }\end{array}$ & $\begin{array}{l}\text { 6.2.E-13 } \\
\text { ND } \\
2.1 \text { E-13 } \\
4.4 \text { E-15 } \\
8.9 \text { E-13 }\end{array}$ & $\begin{array}{c}1.8 \mathrm{E}-04 \\
\mathrm{ND} \\
6.1 \mathrm{E}-05 \\
1.3 \mathrm{E}-06 \\
2.6 \mathrm{E}-04\end{array}$ \\
\hline $\begin{array}{l}296-\mathrm{B}-13 \\
\text { (B Plant/FDH) } \\
{[\mathrm{B} 690]}\end{array}$ & 3.5 & HEPA & $1.2 \mathrm{E}+06$ & $\begin{array}{l}\text { total alpha } \\
\text { total beta }\end{array}$ & $\begin{array}{l}2.3 \mathrm{E}-15 \\
5.8 \mathrm{E}-15\end{array}$ & $\begin{array}{l}2.8 \mathrm{E}-09 \\
7.2 \mathrm{E}-09\end{array}$ \\
\hline $\begin{array}{l}\text { 296-A-13 } \\
\text { (East Tank Farms/FDH) } \\
\text { [E052] }\end{array}$ & 38.1 & HEPA & $0.0 \mathrm{E}+00$ & $\begin{array}{l}\text { total alpha } \\
\text { total beta }\end{array}$ & (did not op & erate) \\
\hline $\begin{array}{l}\text { 296-A-18 } \\
\text { (East Tank Farms/FDH) } \\
\text { [E060] }\end{array}$ & 4.6 & HEPA & $9.6 \mathrm{E}+06$ & $\begin{array}{l}\text { total alpha } \\
\text { total beta }\end{array}$ & $\begin{array}{l}1.2 \mathrm{E}-15 \\
3.8 \mathrm{E}-15\end{array}$ & $\begin{array}{l}1.2 \mathrm{E}-08 \\
3.7 \mathrm{E}-08\end{array}$ \\
\hline
\end{tabular}


Table 2-3

(7 sheets)

\begin{tabular}{|c|c|c|c|c|c|c|}
\hline (3) & \%1 & 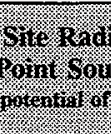 & 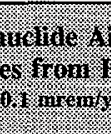 & 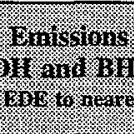 & 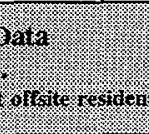 & \\
\hline 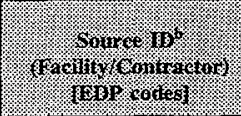 & 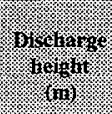 & (3) & 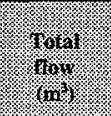 & 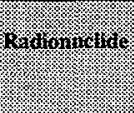 & 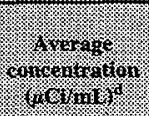 & 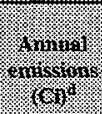 \\
\hline $\begin{array}{l}296-A-19 \\
\text { (East Tank Farms/FDH) } \\
\text { [E061] }\end{array}$ & 4.6 & HEPA & $1.6 \mathrm{E}+07$ & $\begin{array}{l}\text { total alpha } \\
\text { total beta }\end{array}$ & $\begin{array}{l}6.3 \mathrm{E}-16 \\
1.6 \mathrm{E}-15\end{array}$ & $\begin{array}{l}1.0 \mathrm{E}-08 \\
2.7 \mathrm{E}-08\end{array}$ \\
\hline $\begin{array}{l}\text { 296-A-20 } \\
\text { (East Tank Farms/FDH) } \\
\text { [E197] }\end{array}$ & 7.3 & HEPA & $0.0 \mathrm{E}+00$ & $\begin{array}{l}\text { total alpha } \\
\text { total beta }\end{array}$ & \multicolumn{2}{|c|}{ (did not operate) } \\
\hline $\begin{array}{l}296-A-26 \\
\text { (East Tank Farms/FDH) } \\
{[\text { [E297] }}\end{array}$ & 9.4 & HEPA & $2.5 \mathrm{E}+07$ & $\begin{array}{l}\text { total alpha } \\
\text { total beta }\end{array}$ & $\begin{array}{l}3.4 \mathrm{E}-17 \\
1.8 \mathrm{E}-16\end{array}$ & $\begin{array}{l}8.5 \mathrm{E}-10 \\
4.5 \mathrm{E}-09\end{array}$ \\
\hline $\begin{array}{l}296-\mathrm{A}-27 \\
\text { (East Tank Farms/FDH) } \\
{[\mathrm{E} 270, \mathrm{E} 933, \mathrm{E} 934]}\end{array}$ & 3.7 & HEPA & $1.5 \mathrm{E}+07$ & $\begin{array}{l}\text { total alpha } \\
\text { total beta }\end{array}$ & $\begin{array}{l}2.1 \mathrm{E}-16 \\
5.7 \mathrm{E}-15\end{array}$ & $\begin{array}{l}3.2 \mathrm{E}-09 \\
1.4 \mathrm{E}-07\end{array}$ \\
\hline $\begin{array}{l}\text { 296-A-28 } \\
\text { (East Tank Farms/FDH) } \\
\text { [E272] }\end{array}$ & 3.7 & HEPA & $7.8 \mathrm{E}+07$ & $\begin{array}{l}\text { total alpha } \\
\text { total beta }\end{array}$ & $\begin{array}{c}\mathrm{ND} \\
2.8 \mathrm{E}-14\end{array}$ & $\begin{array}{c}\mathrm{ND} \\
2.2 \mathrm{E}-06\end{array}$ \\
\hline $\begin{array}{l}296-\mathrm{A}-29 \\
\text { (East Tank Farms/FDH) } \\
\text { [E901] }\end{array}$ & 3.7 & HEPA & $1.1 \mathrm{E}+07$ & $\begin{array}{l}\text { total alpha } \\
\text { total beta }\end{array}$ & $\begin{array}{l}8.3 \mathrm{E}-17 \\
6.2 \mathrm{E}-14\end{array}$ & $\begin{array}{l}9.1 \mathrm{E}-10 \\
6.8 \mathrm{E}-07\end{array}$ \\
\hline $\begin{array}{l}\text { 296-A-30 } \\
\text { (East Tank Farms/FDH) } \\
\text { [E903] }\end{array}$ & 3.7 & HEPA & $7.4 \mathrm{E}+07$ & $\begin{array}{l}\text { total alpha } \\
\text { total beta }\end{array}$ & $\begin{array}{l}6.9 \mathrm{E}-16 \\
2.9 \mathrm{E}-15\end{array}$ & $\begin{array}{ll}5.1 & \mathrm{E}-08 \\
2.2 & \mathrm{E}-07\end{array}$ \\
\hline $\begin{array}{l}296-A-40 \\
\text { (East Tank Farms/FDH) } \\
\text { [E013,E028,E029] }\end{array}$ & 4.1 & HEPA & $1.4 \mathrm{E}+07$ & $\begin{array}{l}{ }^{90} \mathrm{Sr} \\
{ }^{106} \mathrm{Ru} \\
{ }^{113} \mathrm{Sn} \\
{ }^{125} \mathrm{Sb} \\
{ }^{129} \mathrm{I} \\
{ }^{134} \mathrm{Cs} \\
{ }^{137} \mathrm{Cs} \\
{ }^{238} \mathrm{Pu} \\
{ }^{239.240} \mathrm{Pu} \\
{ }^{241} \mathrm{Am} \\
\text { total alpha } \\
\text { total beta }\end{array}$ & $\begin{array}{c}8.4 \mathrm{E}-17 \\
\text { ND } \\
\text { ND } \\
\text { ND } \\
\text { ND } \\
\text { ND } \\
7.6 \mathrm{E}-17 \\
\text { ND } \\
3.1 \mathrm{E}-17 \\
4.9 \mathrm{E}-17 \\
\mathrm{ND} \\
2.1 \mathrm{E}-15\end{array}$ & $\begin{array}{c}1.1 \mathrm{E}-09 \\
\mathrm{ND} \\
\mathrm{ND} \\
\mathrm{ND} \\
\mathrm{ND} \\
\mathrm{ND} \\
1.0 \mathrm{E}-09 \\
\mathrm{ND} \\
4.2 \mathrm{E}-10 \\
6.8 \mathrm{E}-10 \\
\mathrm{ND} \\
2.9 \mathrm{E}-08\end{array}$ \\
\hline $\begin{array}{l}\text { 296-A-41 } \\
\text { (East Tank Farms/FDH) } \\
\text { [E015] }\end{array}$ & 8.9 & HEPA & $1.2 \mathrm{E}+08$ & $\begin{array}{l}\text { total alpha } \\
\text { total beta }\end{array}$ & $\begin{array}{c}\mathrm{ND} \\
2.1 \mathrm{E}-15\end{array}$ & $\begin{array}{c}\mathrm{ND} \\
2.6 \mathrm{E}-07\end{array}$ \\
\hline
\end{tabular}


Table 2-3

(7 sheets)

\begin{tabular}{|c|c|c|c|c|c|c|}
\hline \multicolumn{7}{|c|}{ 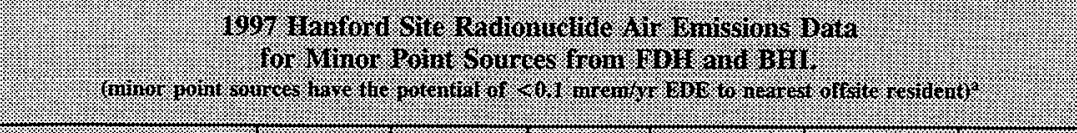 } \\
\hline 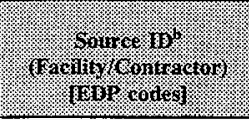 & (1) & 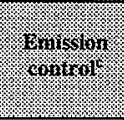 & 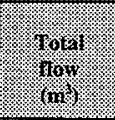 & 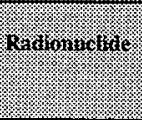 & 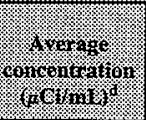 & 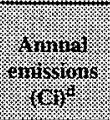 \\
\hline $\begin{array}{l}296-\mathrm{P}-17 \\
\text { (East Tank Farms/FDH) } \\
\text { [E120] }\end{array}$ & 4.6 & HEPA & $0.0 \mathrm{E}+00$ & $\begin{array}{l}\text { total alpha } \\
\text { total beta }\end{array}$ & \multicolumn{2}{|c|}{ (did not operate) } \\
\hline $\begin{array}{l}\text { 296-P-31 } \\
\text { (East Tank Farms/FDH) } \\
\text { [E209] }\end{array}$ & 10.0 & HEPA & $1.6 \mathrm{E}+07$ & $\begin{array}{l}\text { total alpha } \\
\text { total beta }\end{array}$ & $\begin{array}{l}1.8 \mathrm{E}-17 \\
7.7 \mathrm{E}-16\end{array}$ & $\begin{array}{l}2.9 \mathrm{E}-10 \\
1.3 \mathrm{E}-08\end{array}$ \\
\hline $\begin{array}{l}296-\mathrm{P}-33 \\
296-\mathrm{P}-34 \\
\text { (Char. Project/FDH) } \\
\text { [FDH] }\end{array}$ & 4.6 & HEPA & $0.0 \mathrm{E}+00$ & $\begin{array}{l}\text { total alpha } \\
\text { total beta }\end{array}$ & \multicolumn{2}{|c|}{ (did not operate) } \\
\hline $\begin{array}{l}296-\mathrm{A}-21 \\
(242-\mathrm{A} \text { Evaporator/FDH) } \\
{[\mathrm{E} 645]}\end{array}$ & 6.7 & HEPA & $1.8 \mathrm{E}+08$ & $\begin{array}{l}\text { total alpha } \\
\text { total beta }\end{array}$ & $\begin{array}{l}1.9 \mathrm{E}-16 \\
2.2 \mathrm{E}-14\end{array}$ & $\begin{array}{l}3.6 \mathrm{E}-08 \\
4.0 \mathrm{E}-06\end{array}$ \\
\hline $\begin{array}{l}296-\mathrm{E}-1 \\
(\mathrm{ETF} / \mathrm{FDH}) \\
{[\mathrm{E} 036]}\end{array}$ & 15.5 & HEPA & $8.2 E+08$ & $\begin{array}{l}\text { total alpha } \\
\text { total beta }\end{array}$ & $\begin{array}{l}1.3 \mathrm{E}-16 \\
4.9 \mathrm{E}-16\end{array}$ & $\begin{array}{ll}1.1 & \mathrm{E}-07 \\
4.1 \mathrm{E}-07\end{array}$ \\
\hline $\begin{array}{l}296-\mathrm{G}-1 \\
\text { (Grout/FDH) } \\
{[\mathrm{E} 032]}\end{array}$ & 7.6 & HEPA & $0.0 \mathrm{E}+00$ & $\begin{array}{l}\text { total alpha } \\
\text { total beta }\end{array}$ & \multicolumn{2}{|c|}{ (did not operate) } \\
\hline \multicolumn{7}{|c|}{200 West Area Point Sources } \\
\hline $\begin{array}{l}\text { 296-P-22 } \\
\text { (West Tank Farms/FDH) } \\
\text { [W191] }\end{array}$ & 4.6 & HEPA & $1.3 \mathrm{E}+07$ & $\begin{array}{l}\text { total alpha } \\
\text { total beta }\end{array}$ & $\begin{array}{l}3.5 \mathrm{E}-16 \\
4.8 \mathrm{E}-15\end{array}$ & $\begin{array}{l}4.4 \mathrm{E}-09 \\
6.1 \mathrm{E}-08\end{array}$ \\
\hline $\begin{array}{l}296-\mathrm{P}-23 \\
296-\mathrm{P}-28 \text { (backup) } \\
\text { (West Tank Farms/FDH) } \\
\text { [W190,W195] }\end{array}$ & 4.6 & HEPA & $1.3 \mathrm{E}+07$ & $\begin{array}{l}\text { total alpha } \\
\text { total beta }\end{array}$ & $\begin{array}{l}2.0 \text { E-16 } \\
8.2 \text { E-14 }\end{array}$ & $\begin{array}{l}2.7 \mathrm{E}-09 \\
1.1 \mathrm{E}-06\end{array}$ \\
\hline $\begin{array}{l}\text { 296-S-15 } \\
\text { (West Tank Farms/FDH) } \\
\text { [W111] }\end{array}$ & 4.6 & HEPA & $5.1 \mathrm{E}+07$ & $\begin{array}{l}\text { total alpha } \\
\text { total beta }\end{array}$ & $\begin{array}{l}1.8 \mathrm{E}-16 \\
8.7 \mathrm{E}-15\end{array}$ & $\begin{array}{l}9.0 \mathrm{E}-09 \\
4.4 \mathrm{E}-07\end{array}$ \\
\hline $\begin{array}{l}\text { 296-S-18 } \\
\text { (West Tank Farms/FDH) } \\
\text { [W096] }\end{array}$ & 6.7 & HEPA & $1.1 \mathrm{E}+08$ & $\begin{array}{l}\text { total alpha } \\
\text { total beta }\end{array}$ & $\begin{array}{l}1.1 \mathrm{E}-15 \\
3.8 \mathrm{E}-15\end{array}$ & $\begin{array}{l}1.1 \mathrm{E}-07 \\
4.1 \mathrm{E}-07\end{array}$ \\
\hline $\begin{array}{l}\text { 296-T-17 } \\
\text { (West Tank Farms/FDH) } \\
\text { [W117] }\end{array}$ & 10.1 & HEPA & $1.7 \mathrm{E}+07$ & $\begin{array}{l}\text { total alpha } \\
\text { total beta }\end{array}$ & $\begin{array}{c}\mathrm{ND} \\
2.0 \mathrm{E}-15\end{array}$ & $\begin{array}{c}\mathrm{ND} \\
3.4 \mathrm{E}-08\end{array}$ \\
\hline
\end{tabular}


Table 2-3

(7 sheets)

\begin{tabular}{|c|c|c|c|c|c|c|}
\hline 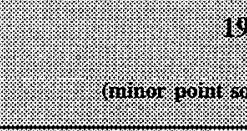 & 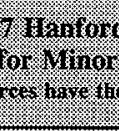 & 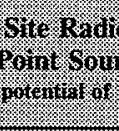 & 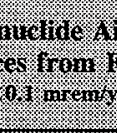 & 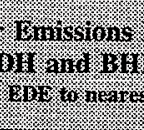 & 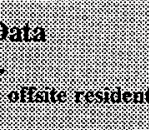 & V. \\
\hline 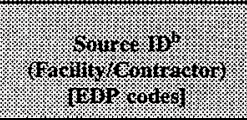 & 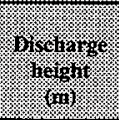 & 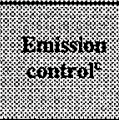 & 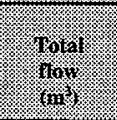 & 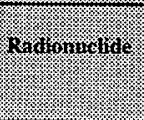 & 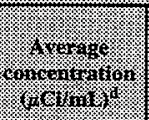 & 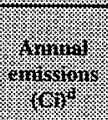 \\
\hline $\begin{array}{l}296-\text { W-3 } \\
\text { (West Tank Farms/FDH) } \\
\text { [W003] }\end{array}$ & 7.6 & HEPA & $4.9 \mathrm{E}+06$ & $\begin{array}{l}\text { total alpha } \\
\text { total beta }\end{array}$ & $\begin{array}{l}6.6 \mathrm{E}-15 \\
9.2 \mathrm{E}-15\end{array}$ & $\begin{array}{l}3.2 \mathrm{E}-08 \\
4.5 \mathrm{E}-08\end{array}$ \\
\hline $\begin{array}{l}291-\mathrm{S}-1 \\
\text { (S Plant/BHI) } \\
\text { [S006] }\end{array}$ & 61.0 & sand filter & $3.0 \mathrm{E}+08$ & $\begin{array}{l}\text { total alpha } \\
\text { total beta }\end{array}$ & $\begin{array}{c}\text { ND } \\
1.5 \mathrm{E}-14\end{array}$ & $\begin{array}{c}\mathrm{ND} \\
4.8 \mathrm{E}-06\end{array}$ \\
\hline $\begin{array}{l}296-\mathrm{S}-2 \\
\text { (S Plant/BHI) } \\
{[\mathrm{S} 032]}\end{array}$ & 20.7 & HEPA & $1.1 \mathrm{E}+07$ & $\begin{array}{l}\text { total alpha } \\
\text { total beta }\end{array}$ & $\begin{array}{l}3.0 \mathrm{E}-15 \\
6.4 \mathrm{E}-15\end{array}$ & $\begin{array}{l}3.3 \mathrm{E}-08 \\
6.9 \mathrm{E}-08\end{array}$ \\
\hline $\begin{array}{l}296-S-7 W \\
\text { 296-S-7E (backup) } \\
\text { (S Plant/BHI) } \\
\text { [S015,S016] }\end{array}$ & 7.6 & HEPA & $1.2 \mathrm{E}+08$ & $\begin{array}{l}\text { total alpha } \\
\text { total beta }\end{array}$ & $\begin{array}{l}2.7 \mathrm{E}-14 \\
6.2 \mathrm{E}-14\end{array}$ & $\begin{array}{l}3.2 \mathrm{E}-06 \\
7.4 \mathrm{E}-06\end{array}$ \\
\hline $\begin{array}{l}291-\mathrm{U}-1 \\
\text { (U Plant/BHI) } \\
\text { [U771] }\end{array}$ & 61.0 & sand filter & $3.9 \mathrm{E}+08$ & $\begin{array}{l}\text { total alpha } \\
\text { total beta }\end{array}$ & $\begin{array}{ll}2.1 & E-15 \\
5.1 & E-13\end{array}$ & $\begin{array}{l}8.1 \mathrm{E}-07 \\
2.0 \mathrm{E}-04\end{array}$ \\
\hline $\begin{array}{l}291-\mathrm{T}-1 \\
(\mathrm{~T} \text { Plant/FDH }) \\
{[\mathrm{T} 785]}\end{array}$ & 61.0 & HEPA & $4.8 \mathrm{E}+08$ & $\begin{array}{l}\text { total alpha } \\
\text { total beta }\end{array}$ & $\begin{array}{l}2.2 \mathrm{E}-14 \\
1.5 \mathrm{E}-13\end{array}$ & $\begin{array}{l}1.0 \mathrm{E}-05 \\
7.5 \mathrm{E}-05\end{array}$ \\
\hline $\begin{array}{l}\text { 296-T-7 } \\
\text { (T Plant/FDH) } \\
\text { [T154] }\end{array}$ & 8.5 & HEPA & $4.4 \mathrm{E}+07$ & $\begin{array}{l}\text { total alpha } \\
\text { total beta }\end{array}$ & $\begin{array}{l}2.6 \mathrm{E}-16 \\
7.6 \mathrm{E}-16\end{array}$ & $\begin{array}{l}1.1 \mathrm{E}-08 \\
3.3 \mathrm{E}-08\end{array}$ \\
\hline $\begin{array}{l}296-\mathrm{T}-13 \\
\text { (T Plant/FDH) } \\
{[\mathrm{T} 786]}\end{array}$ & 20.7 & HEPA & $0.0 \mathrm{E}+00$ & $\begin{array}{l}\text { total aipha } \\
\text { total beta }\end{array}$ & \multicolumn{2}{|c|}{ (did not operate) } \\
\hline $\begin{array}{l}296-\mathrm{T}-11 \\
\text { (TRUSAF/FDH) } \\
\text { [T783] }\end{array}$ & 7.6 & HEPA & $0.0 \mathrm{E}+00$ & $\begin{array}{l}\text { total alpha } \\
\text { total beta }\end{array}$ & \multicolumn{2}{|c|}{ (did not operate) } \\
\hline $\begin{array}{l}296-\mathrm{T}-12 \\
\text { (TRUSAF/FDH) } \\
{[\text { T784] }}\end{array}$ & 7.6 & HEPA & $2.4 \mathrm{E}+08$ & $\begin{array}{l}\text { total alpha } \\
\text { total beta }\end{array}$ & $\begin{array}{l}1.0 \mathrm{E}-15 \\
8.2 \mathrm{E}-15\end{array}$ & $\begin{array}{l}2.4 \mathrm{E}-07 \\
2.0 \mathrm{E}-06\end{array}$ \\
\hline $\begin{array}{l}296-\mathrm{S}-16 \\
(222-\mathrm{S} / \mathrm{FDH}) \\
{[\mathrm{S} 264]}\end{array}$ & 3.0 & HEPA & $2.2 \mathrm{E}+06$ & $\begin{array}{l}\text { total alpha } \\
\text { total beta }\end{array}$ & $\begin{array}{l}3.3 \mathrm{E}-15 \\
1.8 \mathrm{E}-14\end{array}$ & $\begin{array}{l}7.2 \mathrm{E}-09 \\
4.0 \mathrm{E}-08\end{array}$ \\
\hline $\begin{array}{l}\text { 296-S-21 } \\
(222-\mathrm{S} / \mathrm{FDH}) \\
{[\mathrm{S} 289]}\end{array}$ & 11.6 & HEPA & $1.1 \mathrm{E}+09$ & $\begin{array}{l}\text { total alpha } \\
\text { total beta }\end{array}$ & $\begin{array}{c}\text { ND } \\
8.1 \text { E-16 }\end{array}$ & $\begin{array}{c}\mathrm{ND} \\
8.5 \mathrm{E}-07\end{array}$ \\
\hline
\end{tabular}


Table 2-3

(7 sheets)

\begin{tabular}{|c|c|c|c|c|c|c|}
\hline (3. & 6. & 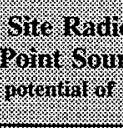 & 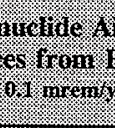 & 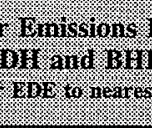 & far & \\
\hline 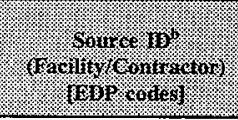 & misciange & 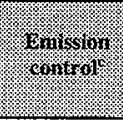 & $\frac{160 \%}{100}$ & 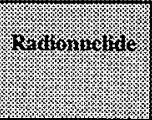 & 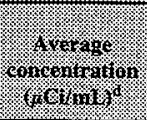 & 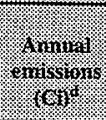 \\
\hline $\begin{array}{l}296-Z-3 \\
\text { (PFP/FDH) } \\
{[\mathrm{Z} 813]}\end{array}$ & 7.6 & HEPA & $1.3 \mathrm{E}+07$ & $\begin{array}{l}{ }^{238} \mathrm{Pu} \\
{ }^{2391240} \mathrm{pu} \\
{ }^{241} \mathrm{Pu} \\
{ }^{241} \mathrm{Am} \\
\text { total alpha } \\
\text { total beta }\end{array}$ & $\begin{array}{ll}1.9 & \text { E-15 } \\
1.7 & \text { E-15 } \\
3.6 & \text { E-15 } \\
8.1 & \text { E-16 } \\
5.2 & \text { E-15 } \\
5.2 & \text { E-15 }\end{array}$ & 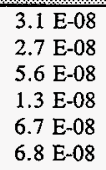 \\
\hline $\begin{array}{l}296-\mathrm{Z}-5 \\
\text { (PFP/FDH) } \\
{[\mathrm{Z} 913]}\end{array}$ & 8.5 & HEPA & $1.1 E+08$ & $\begin{array}{l}\text { total alpha } \\
\text { total beta }\end{array}$ & $\begin{array}{c}\text { ND } \\
1.2 \mathrm{E}-15\end{array}$ & $\begin{array}{c}\text { ND } \\
1.8 \mathrm{E}-07\end{array}$ \\
\hline $\begin{array}{l}296-\mathrm{Z}-6 \\
\text { (PFP/FDH) } \\
{[\mathrm{Z} 802]}\end{array}$ & 4.5 & HEPA & $1.1 \mathrm{E}+08$ & $\begin{array}{l}\text { total alpha } \\
\text { total beta }\end{array}$ & $\begin{array}{c}\mathrm{ND} \\
4.8 \mathrm{E}-15\end{array}$ & $\begin{array}{c}\text { ND } \\
5.2 \mathrm{E}-07\end{array}$ \\
\hline $\begin{array}{l}296-Z-14 \\
(\mathrm{PFP} / F D H) \\
{[\mathrm{Z} 814]}\end{array}$ & 6.1 & HEPA & $2.0 \mathrm{E}+07$ & $\begin{array}{l}\text { total alpha } \\
\text { total beta }\end{array}$ & $\begin{array}{l}2.9 \mathrm{E}-16 \\
2.6 \mathrm{E}-15\end{array}$ & $\begin{array}{l}5.7 \text { E-09 } \\
5.1 \text { E-08 }\end{array}$ \\
\hline $\begin{array}{l}296-Z-15 \\
(\mathrm{PFP} / \mathrm{FDH}) \\
{[\mathrm{Z} 915]}\end{array}$ & 12.8 & HEPA & $2.1 \mathrm{E}+06$ & $\begin{array}{l}\text { total alpha } \\
\text { total beta }\end{array}$ & $\begin{array}{l}2.2 \mathrm{E}-17 \\
2.5 \mathrm{E}-16\end{array}$ & $\begin{array}{l}4.6 \mathrm{E}-1 \mathrm{I} \\
5.0 \mathrm{E}-10\end{array}$ \\
\hline $\begin{array}{l}696-\mathrm{W}-1 \\
\text { (WSCF/FDH) } \\
{[\mathrm{W} 010]}\end{array}$ & 7.6 & HEPA & $7.4 \mathrm{E}+08$ & $\begin{array}{l}\text { total alpha } \\
\text { total beta }\end{array}$ & $\begin{array}{c}\text { ND } \\
7.7 \text { E-17 }\end{array}$ & $\begin{array}{c}\mathrm{ND} \\
5.8 \mathrm{E}-08\end{array}$ \\
\hline $\begin{array}{l}696-\mathrm{W}-2 \\
\text { (WSCF/FDH) } \\
{[\mathrm{W} 011]}\end{array}$ & 9.8 & HEPA & $2.2 \mathrm{E}+07$ & $\begin{array}{l}\text { total alpha } \\
\text { total beta }\end{array}$ & $\begin{array}{c}\text { ND } \\
7.9 \mathrm{E}-16\end{array}$ & $\begin{array}{c}\mathrm{ND} \\
1.8 \mathrm{E}-08\end{array}$ \\
\hline \multicolumn{7}{|c|}{300 Area Point Sources } \\
\hline $\begin{array}{l}\text { 309-PRTR }{ }^{d} \\
\text { (309 Bldg./FDH) }\end{array}$ & 30.5 & HEPA & $7.2 \mathrm{E}+07$ & $\begin{array}{l}\text { total alpha } \\
\text { total beta }\end{array}$ & $\begin{array}{l}\text { ND } \\
\text { ND }\end{array}$ & $\begin{array}{l}\text { ND } \\
\text { ND }\end{array}$ \\
\hline $\begin{array}{l}340-B-B L D G \\
(340 \text { Bldg./FDH }) \\
\text { [F008] }\end{array}$ & 11.6 & HEPA & $9.3 \mathrm{E}+05$ & $\begin{array}{l}\text { total alpha } \\
\text { total beta }\end{array}$ & $\begin{array}{l}\text { ND } \\
\text { ND }\end{array}$ & $\begin{array}{l}\text { ND } \\
\text { ND }\end{array}$ \\
\hline $\begin{array}{l}\text { 340-DECON } \\
\text { (340 Bldg./FDH) } \\
\text { [FO09] }\end{array}$ & 3.0 & $\overline{\text { HEPA }}$ & $1.1 \mathrm{E}+08$ & $\begin{array}{l}\text { total alpha } \\
\text { total beta }\end{array}$ & $\begin{array}{c}\mathrm{ND} \\
4.0 \mathrm{E}-15\end{array}$ & $\begin{array}{c}\mathrm{ND} \\
4.3 \mathrm{E}-07\end{array}$ \\
\hline
\end{tabular}


Table 2-3

\begin{tabular}{|c|c|c|c|c|c|c|}
\hline 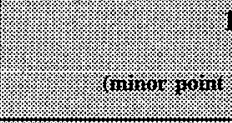 & 6.110 & 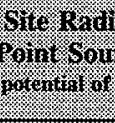 & 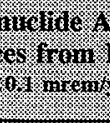 & 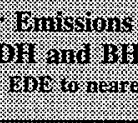 & Hol & \\
\hline 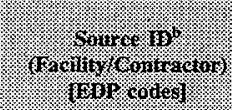 & 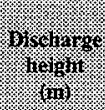 & 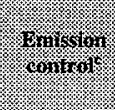 & (1) & M & 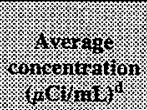 & 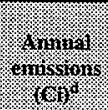 \\
\hline \multicolumn{7}{|c|}{400 Area Point Sources } \\
\hline $\begin{array}{l}\text { FFTF-CB-EX } \\
\text { (FFTF/FDH) } \\
\text { [F011] }\end{array}$ & 14.3 & none & $3.1 \mathrm{E}+08$ & $\begin{array}{l}{ }^{3} \mathrm{H} \text { (as HTO) } \\
{ }^{131} \mathrm{I} \\
\text { total alpha } \\
\text { total beta. }\end{array}$ & $\begin{array}{l}2.5 \mathrm{E}-08 \\
\mathrm{ND} \\
3.6 \mathrm{E}-16 \\
4.8 \mathrm{E}-15\end{array}$ & $\begin{array}{c}7.9 \mathrm{E}+00 \\
\mathrm{ND} \\
1.1 \mathrm{E}-07 \\
1.5 \mathrm{E}-06\end{array}$ \\
\hline $\begin{array}{l}\text { FFTF-RE-SB } \\
\text { (FFTF/FDH) } \\
\text { [F012] }\end{array}$ & 6.1 & none & $1.9 \mathrm{E}+08$ & $\begin{array}{l}\text { 13] I } \\
\text { total alpha } \\
\text { total beta }\end{array}$ & $\begin{array}{c}\text { ND } \\
1.1 \mathrm{E}-15 \\
1.2 \mathrm{E}-14\end{array}$ & $\begin{array}{c}\text { ND } \\
2.0 \mathrm{E}-07 \\
2.1 \mathrm{E}-06\end{array}$ \\
\hline $\begin{array}{l}\text { FFTF-HT-TR } \\
\text { (FFTF/FDH) } \\
\text { [F013] }\end{array}$ & 8.8 & none & $8.0 \mathrm{E}+07$ & $\begin{array}{l}\text { total alpha } \\
\text { total beta }\end{array}$ & $\begin{array}{ll}2.1 & \text { E-16 } \\
2.7 & \text { E-15 }\end{array}$ & $\begin{array}{ll}1.7 & \mathrm{E}-08 \\
2.2 & \mathrm{E}-07\end{array}$ \\
\hline $\begin{array}{l}\text { 437-MN\&ST } \\
\text { (MASF/FDH) } \\
\text { [F014] }\end{array}$ & 9.1 & HEPA & $2.3 \mathrm{E}+08$ & $\begin{array}{l}\text { total alpha } \\
\text { total beta }\end{array}$ & $\begin{array}{l}2.5 \mathrm{E}-16 \\
2.6 \mathrm{E}-15\end{array}$ & $\begin{array}{l}5.5 \mathrm{E}-08 \\
5.9 \mathrm{E}-07\end{array}$ \\
\hline $\begin{array}{l}437-1-61 \\
\text { (MASF/FDH) } \\
\text { [F019] }\end{array}$ & 11.7 & HEPA & $2.3 E+08$ & $\begin{array}{l}\text { total alpha } \\
\text { total beta }\end{array}$ & $\begin{array}{c}\text { ND } \\
4.0 \mathrm{E}-16\end{array}$ & $\begin{array}{c}\text { ND } \\
9.0 \mathrm{E}-08\end{array}$ \\
\hline
\end{tabular}

Notes:

a $\mathrm{EDE}=$ effective dose equivalent.

b ID = identification, i.e., the alpha-numeric designator for the respective point source; EDP code = electronic data processing code for sampler identification; FDH = Fluor Daniel Hanford, Inc.; BHI = Bechtel Hanford, Inc.

c Efficiencies are: $\geq 99.95 \%$ for HEPA; $\geq 95 \%$ for charcoal; $\geq 99.8 \%$ for sand filter; $0 \%$ for no emission control; HEPA $=$ high efficiency particulate air filter.

d $1 \mu \mathrm{Ci} / \mathrm{mL}=3.7 \mathrm{E}+10 \mathrm{~Bq} / \mathrm{mL} ; 1$ curie $=3.7 \mathrm{E}+10$ becquerel; $\mathrm{ND}=$ none detected (i.e. either the radionuclide was not detected in any sample during the year, or the average of all the measurements for that given radionuclide or type of radioactivity made during the year was below background levels). 
Table 2-4

(3 sheets)

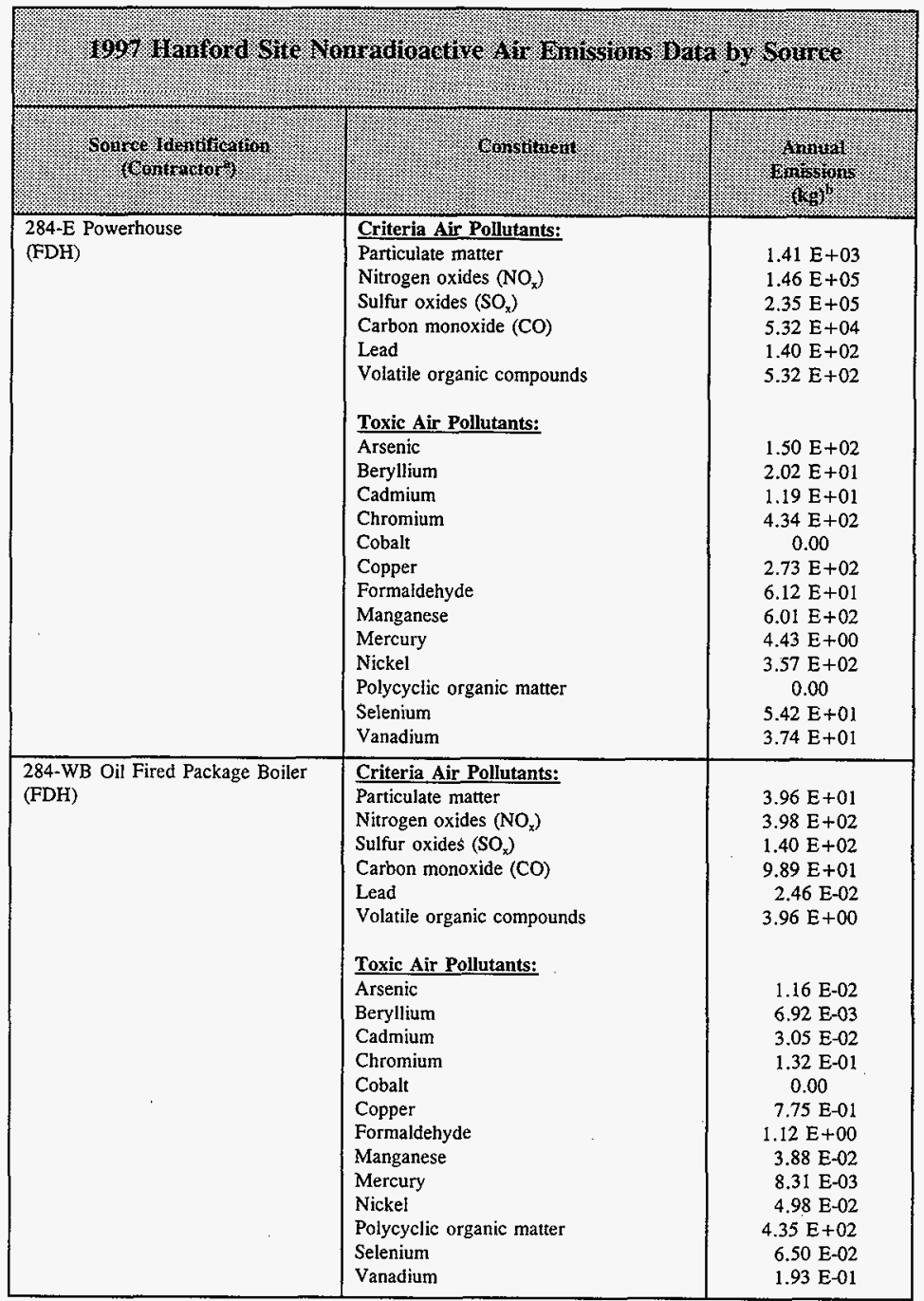


Table 2-4

(3 sheets)

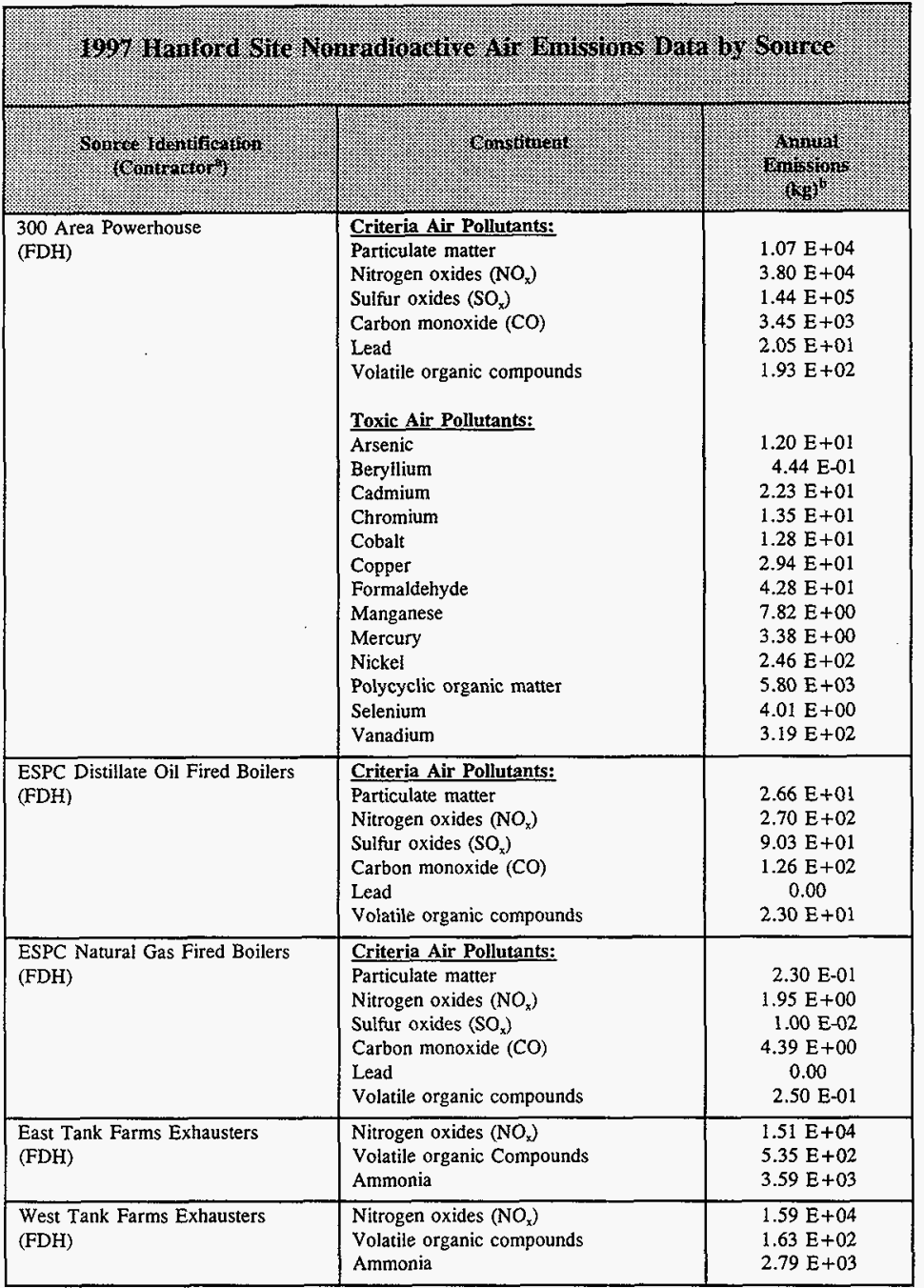


HNF-EP-0527-7

Table 2-4

(3 sheets)

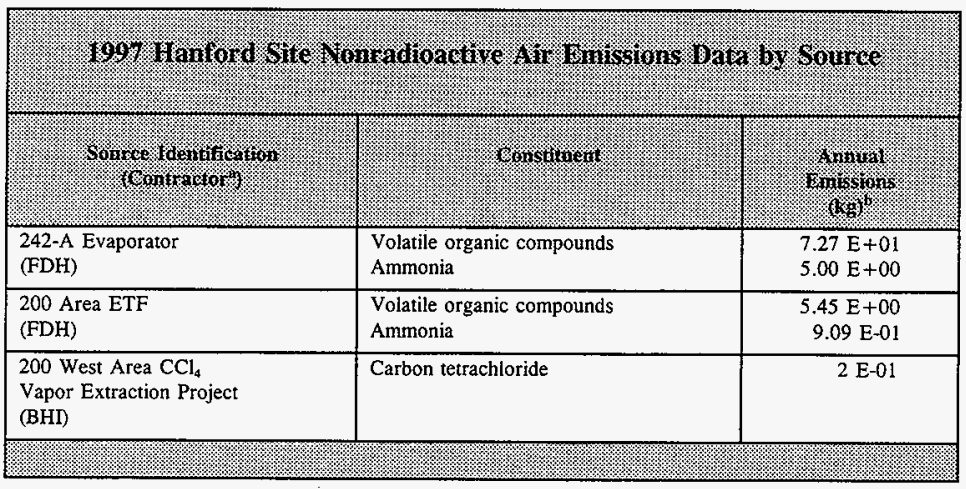

Notes:

a $\mathrm{FDH}=$ Fluor Daniel Hanford, Inc.; $\mathrm{BHI}=$ Bechtel Hanford, Inc.

b Powerhouse emissions calculated using EPA emission factors (EPA 450/4-90-003) and based on total fuel consumption. 
HNF-EP-0527-7

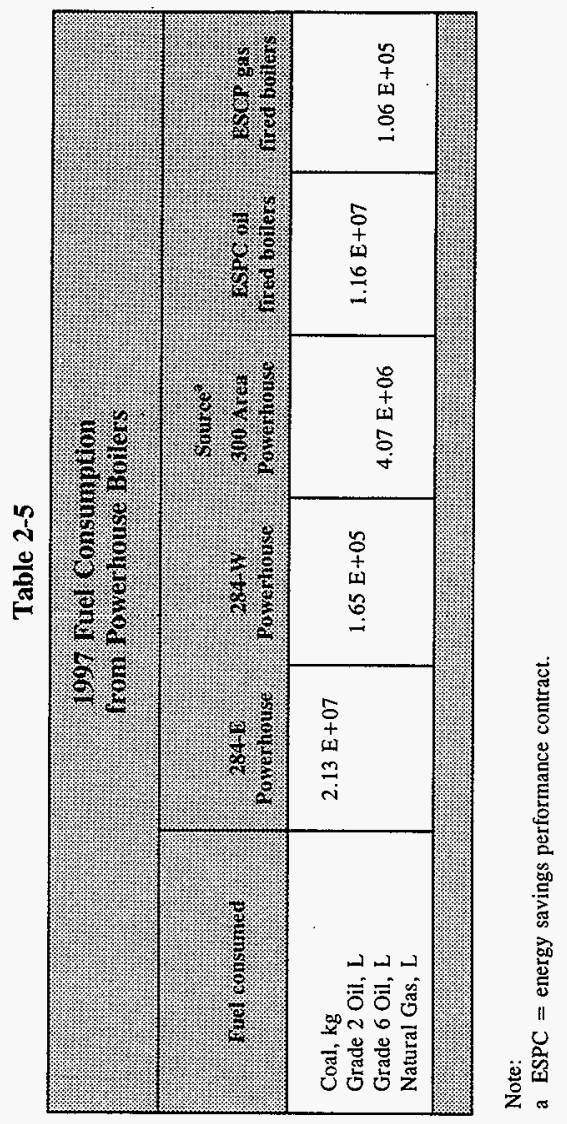




\subsection{LIQUID EFFLUENTS}

The majority of liquid effluents released to the environment from facilities and activities managed by FDH and BHI were discharged under the appropriate state and federal discharge permits. Data on the 1997 radioactive and nonradioactive liquid effluents are presented in this section.

By the end of June 1995, several liquid effluent streams were either discontinued or rerouted through the 200 Area TEDF, eliminating these as individual liquid effluent streams. The streams rerouted included the Plutonium Finishing Plant waste water, 222-S Laboratory steam condensate, T Plant waste water, 284-W Power Plant waste water, PUREX Plant waste water, B Plant waste water, and 242-A-81 waste water streams. As a result, these streams no longer discharge directly to the environment and no longer require reporting as effluent streams.

On August 8, 1997, the remaining liquid effluent streams discharging to the 216-B-3 Pond (C Lobe) were permanently rerouted to the 200 Area TEDF, eliminating these as individual liquid effluent streams. The streams rerouted included the 242-A Evaporator cooling water, 242-A Evaporator steam condensate, 241-A Tank Farm cooling water, 244-AR Vault cooling water, 284-E Power Plant waste water, and B Plant cooling water streams. After 1997, these streams no longer discharge directly to the environment and will no longer require reporting as effluent streams.

\subsection{NPDES PERMITTED DISCHARGES TO COLUMBIA RIVER}

Liquid effluents discharged to the Columbia River from the $100 \mathrm{~N}, 100 \mathrm{~K}$, and 300 Areas are regulated by the parameters in the NPDES permits (WA-000374-3, WA-002591-7, and WA-R-10-000F) for the Hanford Site. A list of the NPDES permitted discharge points and is provided in Table 3-1. Summaries of the 1997 discharge monitoring reports (DMRs), which are used to demonstrate compliance with the NPDES permits, are provided in Table 3-2.

The measured quantities of radionuclides discharged in liquid effluents to the Columbia River are summarized in the Executive Summary, Table ES-2. Releases of specific radionuclides and total activity discharged by individual liquid effluent streams are presented in Table 3-3.

The following sections discuss the NPDES permitted discharge streams active during 1997. 


\subsubsection{8-K Outfall}

1908-K Outfall, Discharge Number 004, discharges potentially hazardous chemical and radioactive substances to the Columbia River. The outfall is routinely monitored for flow, temperature, $\mathrm{pH}$, total suspended solids, and chlorine. The outfall is also routinely monitored for the following radionuclides: ${ }^{3} \mathrm{H},{ }^{90} \mathrm{Sr},{ }^{238} \mathrm{Pu},{ }^{239 / 240} \mathrm{Pu},{ }^{241} \mathrm{Am}$, gamma emitting radionuclides, total alpha activity, and total beta activity.

\subsubsection{N-Springs}

$\mathrm{N}$-Springs, Discharge Number 1301, discharges potentially hazardous chemical and radioactive substances to the Columbia River. The outfall is routinely monitored for $\mathrm{pH}$, oil and grease, chromium, iron, and nitrogen. N-Springs are also routinely monitored for ${ }^{3} \mathrm{H}$ and ${ }^{90} \mathrm{Sr}$.

\subsubsection{Area TEDF}

300 Area TEDF, Discharge Number 001A, discharges treated waste water, under NPDES permit WA-002591-7. The outfall is routinely monitored for numerous constituents, which are listed in Table 3-2.

All nonradioactive liquid effluents from the 300 Area are discharged to the 300 Area TEDF for treatment prior to discharge. 300 Area TEDF discharged $6.2 \mathrm{E}+08 \mathrm{~L}$ $(1.7 \mathrm{E}+08 \mathrm{Gal})$ of nonhazardous nonradioactive liquid wastes to the Columbia River during 1997. All radioactive liquid effluents from the 300 Area were transported to the TanK Farms in the 200 East Area, via the 340 Facility, located in the 300 Area.

\subsection{STATE PERMITTED DISCHARGES TO THE SOIL}

During 1997, all liquid effluent streams discharging to the soil column were either covered by a state waste discharge permit or a permit application was submitted to permit the discharge, unless exempted under CERCLA or regulatory agreements with Ecology. Most of the liquid effluent streams preceded regulation by the state. The Hanford Site has made a significant effort to bring all of the liquid effluent streams into compliance with state regulations. Liquid effluent streams with state waste discharge permits is provided in Table 3-1. 


\subsubsection{Area TEDF}

200 Area TEDF discharges waste water, under state waste discharge permit ST 4502. The outfall is routinely monitored for a multitude of constituents, which are all listed in Table 3-4.

\subsubsection{Area ETF}

200 Area ETF discharges treated waste water, under state waste discharge permit ST 4500 . The outfall is routinely monitored for numerous chemical and radioactive constituents, which are listed in Tables 3-3 and 3-4.

\subsubsection{Area Secondary Cooling Water}

400 Area Secondary Cooling Water Stream discharges cooling water from the secondary cooling loop of the FFTF reactor, under state waste discharge permit ST 4503 . The cooling water discharges meet the drinking water standard. The discharge point is routinely monitored for flow, $\mathrm{pH}$, nitrate, nitrite, arsenic, chloride, cyanide, manganese, phosphorus, total dissolved solids, total organic halides, total beta activity, and tritium.

\subsubsection{3-N Backwash Discharge Pond}

Waste water discharges going to the 183-N Backwash Discharge Pond is associated with 183-N Water Treatment Facility activities, and are performed under state waste discharge permit ST 4503. The 183-N Water Treatment Facility converts raw water, from the Columbia River, into potable water for the $100-\mathrm{N}$ Area. Three waste water streams are discharged to the 183-N Backwash Discharge Pond without treatment. These streams contain waste water from the annual draining and washing of the coagulator basins, a continuously flowing sample tap and a water container drying area drain, and from backwashing the multimedia gravity filters. The discharge site is routinely monitored for flow rate, $\mathrm{pH}$, sulfate, trihalomethanes, and residual chlorine.

\subsubsection{0-N Sewage Lagoon}

$100-\mathrm{N}$ Sewage Lagoon treats sewage from the $100-\mathrm{N}$ and 200 Areas. Domestic waste water is discharged from the 100-N Sewage Lagoon, under state waste discharge permit ST 4507. The discharge of leachate, from the residual solids, and radioactive waste is not permitted for this discharge site. The discharge site is routinely monitored for influent flow, effluent flow, $\mathrm{pH}$, biochemical oxygen demand, total suspended solids, and total dissolved solids. 


\subsubsection{Hydrotesting, Maintenance, and Construction Discharges}

Waste water discharges as a result of hydrotesting, maintenance, and construction activities are performed under state waste discharge permit ST 4508. These discharges occur at numerous locations throughout the Hanford Site and only require monitoring and reporting for significant discharges.

\subsubsection{Cooling Water and Steam Condensate Discharges}

Cooling water and steam condensate discharges are performed under state waste discharge permit ST 4509. These discharges occur at numerous locations throughout the Hanford Site and do not require routine monitoring and reporting.

\subsubsection{Storm Water Discharges}

A permit application for storm water discharges has been submitted to Ecology, but a permit has not been issued at the time of this report. Storm water discharges occur at numerous locations throughout the Hanford Site and are not routinely monitored and reported.

\subsection{SANITARY SEWAGE DISCHARGES TO THE SOIL}

Various facilities discharged sanitary sewage. In the $100-\mathrm{N}$ Area, sanitary waste water was discharged to the 100-N Sewage Lagoon and five septic tanks. In the 100-B, 100-D, 100-H, and 100-K Areas, sanitary sewage was discharged to septic tanks and drain fields. In the 200 Areas, sanitary waste water was discharged to a system of septic tanks and drain fields. Sludge was pumped from septic tanks in the 200 Areas and taken to the 100-N Sewage Lagoon for disposal. In the 300 Area, sanitary sewage was discharged to the city of Richland's POTW. In the 400 Area, sanitary sewage was discharged to a sewage treatment plant and lagoon until Aptil 15, 1997, when discharges started going to the Washington Public Power Supply's sewage treatment plant.

The estimated volume of sewage discharged by operating area during 1997 is shown in Table 3-5. All sanitary sewer discharges are estimated by multiplying the total number of personnel stationed in each area by $95 \mathrm{~L} /$ day-person ( 25 gal/day-person) and by the 251 business days in 1997 . 
Table 3-1

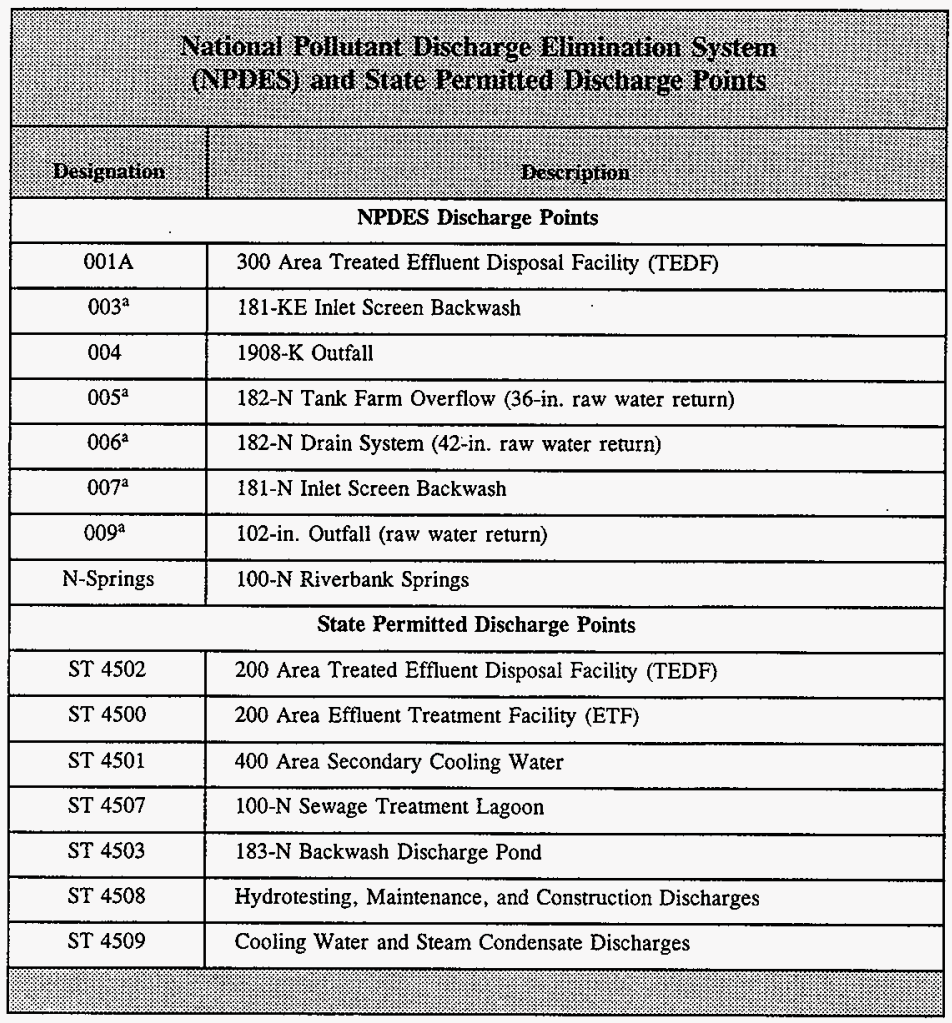

Note:

a There were no discharges for this point during 1997. 
Table 3-2

(2 sheets)

\begin{tabular}{|c|c|c|c|c|c|c|}
\hline 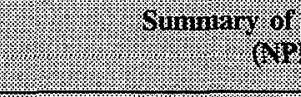 & axis & Wuma & ofor & (3) & 2 & \\
\hline 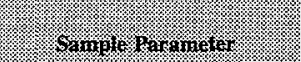 & 細 & Wx & 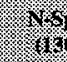 & 1) & 3010 & WO10 \\
\hline & 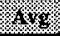 & (1) & \% & 到 & \% & ytax \\
\hline Flow rate (MGD) & 0.79 & 4.52 & * & * & 0.266 & 0.374 \\
\hline Temperature $\left({ }^{\circ} \mathrm{F}\right)$ & $*$ & 73.0 & * & 59.2 & 79.1 & 93.6 \\
\hline $\mathrm{pH}$ (minimum and maximum) & 6.9 & 7.6 & 7.1 & 7.7 & 6.3 & 9.0 \\
\hline Total suspended solids $(\mu \mathrm{g} / \mathrm{L})$ & 2.3 & 4.0 & * & * & 1300 & 6000 \\
\hline Oil and grease ( $\mathrm{mg} / \mathrm{L}$ ) & * & * & 4.4 & 11.1 & * & * \\
\hline Aluminum $(\mu \mathrm{g} / \mathrm{L})$ & * & $*$ & * & * & 14.5 & 53.0 \\
\hline Arsenic $(\mu \mathrm{g} / \mathrm{L})$ & * & * & * & * & $<0.4$ & $<0.4$ \\
\hline Beryllium $(\mu \mathrm{g} / \mathrm{L})$ & $*$ & * & * & * & $<0.2$ & 0.2 \\
\hline Cadmium $(\mu \mathrm{g} / \mathrm{L})$ & * & * & * & * & $<0.2$ & $<0.2$ \\
\hline Chromium (mg/L) & * & * & 0.002 & 0.003 & * & $*$ \\
\hline Chlorine $(\mathrm{mg} / \mathrm{L})$ & 0.03 & 0.08 & $*$ & * & * & * \\
\hline Copper $(\mu \mathrm{g} / \mathrm{L})$ & * & * & * & * & 2.6 & 3.8 \\
\hline Iron $(\mu \mathrm{g} / \mathrm{L})$ & * & * & 0.02 & 0.03 & 12.7 & 54.4 \\
\hline Lead $(\mu \mathrm{g} / \mathrm{L})$ & * & * & $*$ & * & $<0.2$ & $<0.2$ \\
\hline Manganese $(\mu \mathrm{g} / \mathrm{L})$ & * & * & $*$ & * & $<0.4$ & 0.9 \\
\hline Mercury $(\mu \mathrm{g} / \mathrm{L})$ & $*$ & * & $*$ & *. & $<0.2$ & $<0.2$ \\
\hline Nickel $(\mu \mathrm{g} / \mathrm{L})$ & * & * & $*$ & * & $<0.7$ & 2.3 \\
\hline Radium (pCi/L) & * & * & * & * & $<0.23$ & 0.09 \\
\hline Selenium $(\mu \mathrm{g} / \mathrm{L})$ & * & $*$ & * & * & $<3$ & $<3$ \\
\hline Silver $(\mu \mathrm{g} / \mathrm{L})$ & $*$ & * & * & $*$ & $<0.3$ & $<0.3$ \\
\hline Zinc $(\mu \mathrm{g} / \mathrm{L})$ & * & * & $*$ & $*$ & 5.3 & 26.8 \\
\hline Nitrogen (as ammonia) $(\mu \mathrm{g} / \mathrm{L})$ & * & * & 0.06 & 0.07 & $<56.1$ & 260.0 \\
\hline Bis (2-ethylhexyl) phthalate ( $\mu \mathrm{g} / \mathrm{L})$ & * & * & $*$ & * & $<4.8$ & 20.0 \\
\hline Chlorodiflouromethane $(\mu \mathrm{g} / \mathrm{L})$ & * & * & * & * & $<0.1$ & $<0.1$ \\
\hline Chloroform $(\mu \mathrm{g} / \mathrm{L})$ & * & $*$ & $*$ & * & $<4.9$ & 7.0 \\
\hline Coliform (growth/100mL) & $*$ & * & $*$ & * & $<3.70$ & $<3.70$ \\
\hline Cyanide $(\mu \mathrm{g} / \mathrm{L})$ & * & * & * & * & $<4.8$ & 5.2 \\
\hline Dichlorobromomethane $(\mu \mathrm{g} / \mathrm{L})$ & $*$ & * & * & $*$ & $<2.2$ & $<2.2$ \\
\hline
\end{tabular}


HNF-EP-0527-7

Table 3-2

(2 sheets)

\begin{tabular}{|c|c|c|c|c|c|c|}
\hline 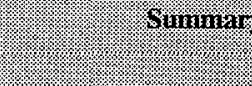 & $\frac{3 y n}{4}$ & oxta & Bix & 3. & em & \\
\hline 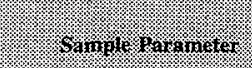 & 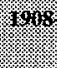 & Wu & 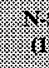 & \% & Wor & WII \\
\hline & xis & \%) & 4 & 10 & 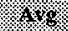 & 16as \\
\hline 1,1-Dichloroethane $(\mu \mathrm{g} / \mathrm{L})$ & $*$ & * & $*$ & * & $<4.7$ & $<4.7$ \\
\hline Methylene chloride $(\mu \mathrm{g} / \mathrm{L})$ & * & * & * & * & $<3$ & $<3$ \\
\hline Nitrite $\left(\mathrm{NO}_{2}\right)(\mu \mathrm{g} / \mathrm{L})$ & * & * & $*$ & * & $<69.3$ & 216.0 \\
\hline Tetrachloroethylene $(\mu \mathrm{g} / \mathrm{L})$ & * & * & * & * & $<5$ & $<5$ \\
\hline 1,1,1-Trichloroethane $(\mu \mathrm{g} / \mathrm{L})$ & * & * & * & * & $<5.0$ & $<5.0$ \\
\hline Trichloroethylene $(\mu \mathrm{g} / \mathrm{L})$ & * & * & * & * & $<1.9$ & $<1.9$ \\
\hline Toluene $(\mu \mathrm{g} / \mathrm{L})$ & * & * & * & * & $<6.0$ & $<6.0$ \\
\hline
\end{tabular}

Note:

a $\mathrm{MGD}=$ million gallons per day; * = analysis not required. 
Table 3-3

(3 sheets)

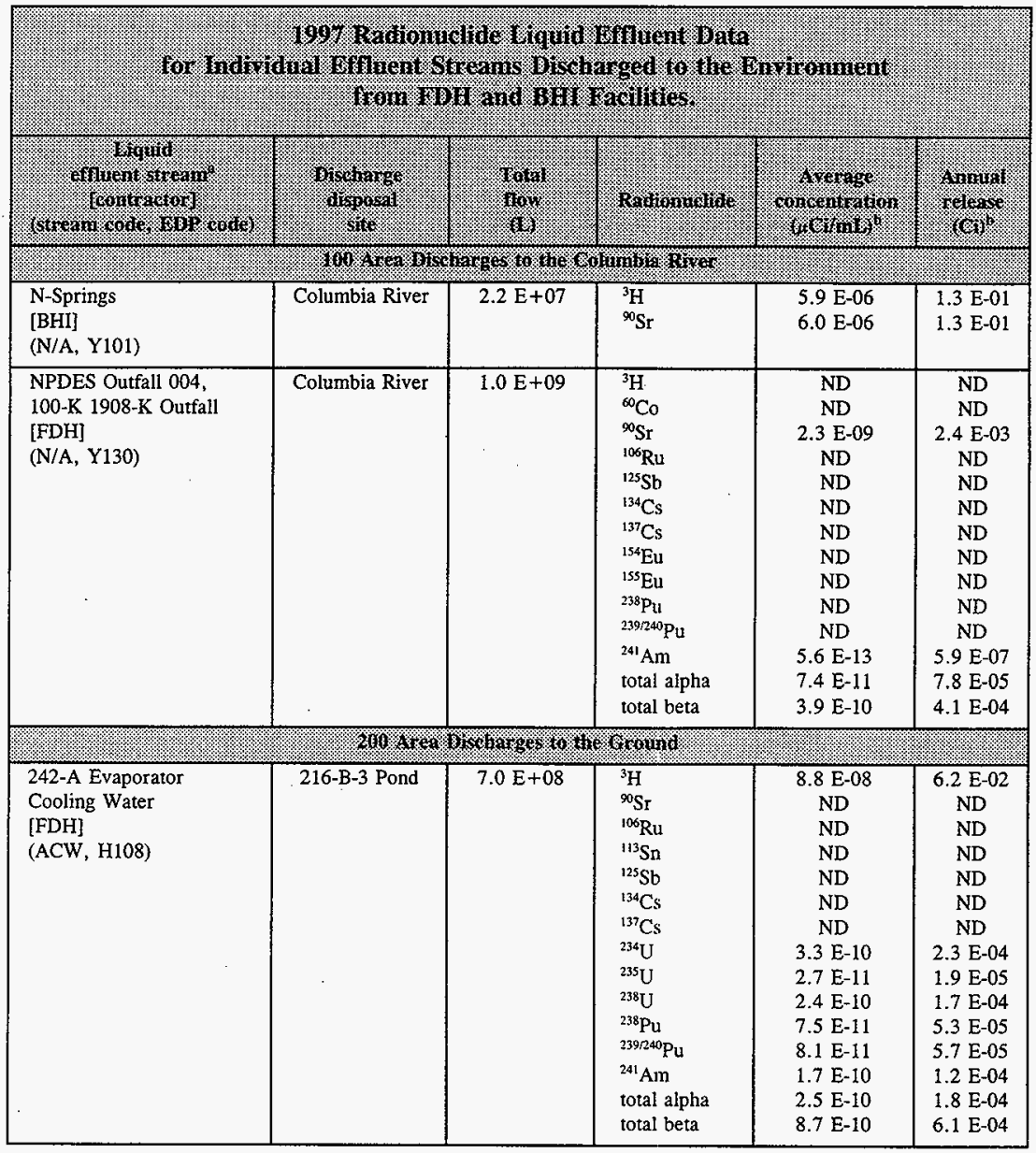


Table 3-3

(3 sheets)

\begin{tabular}{|c|c|c|c|c|c|}
\hline \multicolumn{6}{|c|}{ 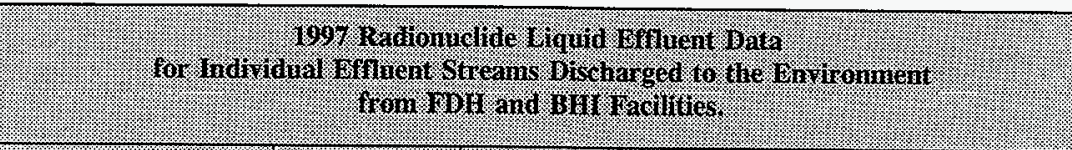 } \\
\hline 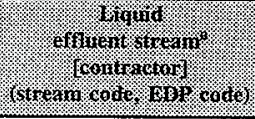 & miscirager & Wow & 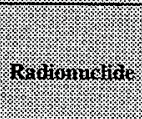 & 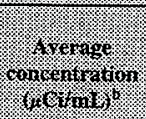 & 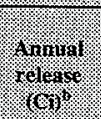 \\
\hline $\begin{array}{l}\text { 242-A Evaporator } \\
\text { Steam Condensate } \\
\text { [FDH] } \\
\text { (ASC, H110) }\end{array}$ & 216-B-3 Pond & $3.2 \mathrm{E}+06$ & $\begin{array}{l}{ }^{3} \mathrm{H} \\
{ }_{90}^{90} \mathrm{Sr} \\
{ }^{106} \mathrm{Ru} \\
{ }^{113} \mathrm{Sn} \\
{ }^{125} \mathrm{Sb} \\
{ }^{134} \mathrm{Cs} \\
{ }^{137} \mathrm{Cs} \\
{ }^{238} \mathrm{Pu} \\
{ }^{239 / 240} \mathrm{Pu} \\
{ }^{241} \mathrm{Am} \\
\text { total alpha } \\
\text { total beta }\end{array}$ & $\begin{array}{c}\text { ND } \\
4.9 \mathrm{E}-10 \\
\text { ND } \\
\text { ND } \\
\text { ND } \\
\text { ND } \\
\text { ND } \\
\text { ND } \\
4.7 \text { E-11 } \\
1.5 \text { E-10 } \\
\text { ND } \\
1.8 \text { E-09 }\end{array}$ & $\begin{array}{c}\text { ND } \\
\text { 8.6 E-06 } \\
\text { ND } \\
\text { ND } \\
\text { ND } \\
\text { ND } \\
\text { ND } \\
\text { ND } \\
1.5 \mathrm{E}-07 \\
4.8 \mathrm{E}-07 \\
\text { ND } \\
5.9 \mathrm{E}-06\end{array}$ \\
\hline $\begin{array}{l}\text { 241-A Tank Farm } \\
\text { Cooling Water } \\
\text { [FDH] } \\
(\mathrm{CA} 8, \mathrm{H} 115)\end{array}$ & 216-B-3 Pond & $5.4 \mathrm{E}+08$ & $\begin{array}{l}{ }^{90} \mathrm{Sr} \\
{ }^{106} \mathrm{Ru} \\
{ }^{113} \mathrm{Sn} \\
{ }^{125} \mathrm{Sb} \\
{ }^{134} \mathrm{Cs} \\
{ }^{137} \mathrm{Cs} \\
{ }^{238} \mathrm{Pu} \\
{ }^{239} 9240 \mathrm{Pu} \\
{ }^{241} \mathrm{Am} \\
\text { total alpha } \\
\text { total beta }\end{array}$ & $\begin{array}{c}2.0 \mathrm{E}-10 \\
\mathrm{ND} \\
\mathrm{ND} \\
\mathrm{ND} \\
\mathrm{ND} \\
\mathrm{ND} \\
2.3 \mathrm{E}-11 \\
1.8 \mathrm{E}-11 \\
1.1 \mathrm{E}-10 \\
3.7 \mathrm{E}-10 \\
2.7 \mathrm{E}-10\end{array}$ & $\begin{array}{c}1.1 \mathrm{E}-04 \\
\mathrm{ND} \\
\mathrm{ND} \\
\mathrm{ND} \\
\mathrm{ND} \\
\mathrm{ND} \\
1.3 \mathrm{E}-05 \\
9.7 \mathrm{E}-06 \\
5.9 \mathrm{E}-05 \\
2.0 \mathrm{E}-04 \\
1.5 \mathrm{E}-04\end{array}$ \\
\hline $\begin{array}{l}\text { 244-AR Vault } \\
\text { Cooling Water } \\
\text { [FDH] } \\
\text { (CAR, H116) }\end{array}$ & 216-B-3 Pond & $2.0 \mathrm{E}+06$ & $\begin{array}{l}{ }^{90} \mathrm{Sr} \\
{ }^{106} \mathrm{Ru} \\
{ }^{113} \mathrm{Sn} \\
{ }^{125} \mathrm{Sb} \\
{ }^{134} \mathrm{Cs} \\
{ }^{137} \mathrm{Cs} \\
{ }^{238} \mathrm{Pu} \\
{ }^{239 / 240} \mathrm{Pu} \\
{ }^{241} \mathrm{Am} \\
\text { total alpha } \\
\text { total beta }\end{array}$ & $\begin{array}{c}9.6 \mathrm{E}-10 \\
\mathrm{ND} \\
\mathrm{ND} \\
\mathrm{ND} \\
\mathrm{ND} \\
\mathrm{ND} \\
2.0 \mathrm{E}-11 \\
2.0 \mathrm{E}-11 \\
1.1 \mathrm{E}-10 \\
\mathrm{ND} \\
2.4 \mathrm{E}-09\end{array}$ & $\begin{array}{c}1.2 \mathrm{E}-06 \\
\mathrm{ND} \\
\mathrm{ND} \\
\mathrm{ND} \\
\mathrm{ND} \\
\mathrm{ND} \\
2.5 \mathrm{E}-08 \\
2.5 \mathrm{E}-08 \\
1.3 \mathrm{E}-07 \\
\mathrm{ND} \\
3.0 \mathrm{E}-06\end{array}$ \\
\hline $\begin{array}{l}\text { B-Plant Cooling Water } \\
\text { [FDH] } \\
\text { (CBC, H117) }\end{array}$ & 216-B-3 Pond & $1.4 \mathrm{E}+07$ & $\begin{array}{l}{ }^{90} \mathrm{Sr} \\
{ }^{106} \mathrm{Ru} \\
{ }^{113} \mathrm{Sn} \\
{ }^{125} \mathrm{Sb} \\
{ }^{134} \mathrm{Cs} \\
{ }^{137} \mathrm{Cs} \\
\text { total alpha } \\
\text { total beta }\end{array}$ & $\begin{array}{c}\text { ND } \\
\text { ND } \\
\text { ND } \\
\text { ND } \\
\text { ND } \\
3.5 \text { E-10 } \\
5.1 \text { E-11 } \\
4.1 \text { E-09 }\end{array}$ & $\begin{array}{c}\text { ND } \\
\text { ND } \\
\text { ND } \\
\text { ND } \\
\text { ND } \\
5.0 \mathrm{E}-06 \\
7.3 \mathrm{E}-07 \\
5.8 \mathrm{E}-05\end{array}$ \\
\hline
\end{tabular}


Table 3-3

(3 sheets)

\begin{tabular}{|l|lll}
\hline \\
\hline
\end{tabular}

Notes:

a $\mathrm{FDH}=$ Fluor Daniel Hanford, Inc.; BHI = Bechtel Hanford, Inc; EDP Code = Electronic Data Processing Code.

b $1 \mu \mathrm{Ci} / \mathrm{mL}=3.7 \mathrm{E}+10 \mathrm{~Bq} / \mathrm{m}^{3} ; 1$ curie $=3.7 \mathrm{E}+10$ becquerel; $\mathrm{ND}=$ none detected. 
Table 3-4

(4 sheets)

\begin{tabular}{|c|c|c|c|c|c|c|c|c|c|c|}
\hline 3.1. & 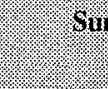 & mars of & $\begin{array}{l}300 \% \\
\text { sorat }\end{array}$ & 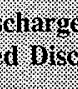 & Wols & 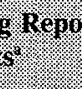 & & & & \\
\hline 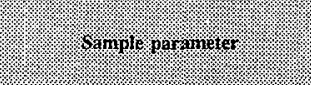 & \%ox & 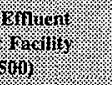 & \%olom & 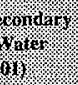 & \%200 & 10y. & mos & 13. & 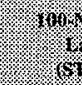 & mor \\
\hline & (1) & 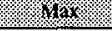 & 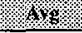 & 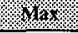 & $x$ & 1) & 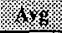 & $x$ & X & (4) \\
\hline Effluent flow rate (gal/month) & $1.27 \mathrm{E}+06$ & $3.25 \mathbf{E}+06$ & * & $*$ & * & * & * & * & $*$ & * \\
\hline Effluent flow rate (gal/day) & * & * & * & $*$ & $*$ & $*$ & 6,180 & 92,580 & 2,000 & 7,668 \\
\hline Effluent flow rate (gal/min) & * & * & 25.4 & 158.0 & 448 & 2,294 & * & * & * & * \\
\hline Influent flow rate (gal/day) & * & * & * & * & * & * & * & * & 8,395 & 10,733 \\
\hline $\mathrm{pH}$ (minimum and maximum) & * & * & 8.16 & 8.70 & 6.20 & 9.71 & 6.33 & 6.69 & 7.1 & 7.7 \\
\hline Conductivity $(\mu \mathrm{mhos} / \mathrm{cm})$ & NQ & NQ & $*$ & $*$ & 171 & 231 & * & * & * & * \\
\hline Total suspended solids $(\mu \mathrm{g} / \mathrm{L})$ & NQ & NQ & $*$ & * & 1200 & 11000 & * & * & 31,000 & 48,000 \\
\hline Total dissolved solids $(\mu \mathrm{g} / \mathrm{L})$ & NQ & NQ & 480,571 & 548,000 & 82,558 & 105,750 & * & * & 252,000 & 310,000 \\
\hline Biochemical oxygen demand (mg/L) & * & $*$ & $*$ & * & $*$ & * & * & * & 32 & 107 \\
\hline Total organic carbon ( $\mu \mathrm{g} / \mathrm{L})$ & NQ & NQ & $*$ & $*$ & * & * & * & * & * & * \\
\hline Total organic halides $(\mu \mathrm{g} / \mathrm{L})$ & * & * & 59.3 & 146.0 & * & * & * & * & * & * \\
\hline Total trihalomethanes $(\mu \mathrm{Ci} / \mathrm{L})$ & $*$ & $*$ & * & * & NQ & NQ & $*$ & * & $*$ & * \\
\hline Oil and grease $(\mathrm{mg} / \mathrm{L})$ & * & $*$ & $*$ & * & NQ & NQ & $*$ & * & * & * \\
\hline Arsenic $(\mu \dot{\mathrm{g}} / \mathrm{L})$ & NQ & NQ & NQ & NQ & NQ & NQ & * & * & * & * \\
\hline Beryllium $(\mu \mathrm{g} / \mathrm{L})$ & NQ & NQ & * & * & * & * & * & * & * & * \\
\hline Cadmium $(\mu \mathrm{g} / \mathrm{L})$ & NQ & NQ & NQ & NQ & NQ & NQ & * & * & * & * \\
\hline
\end{tabular}




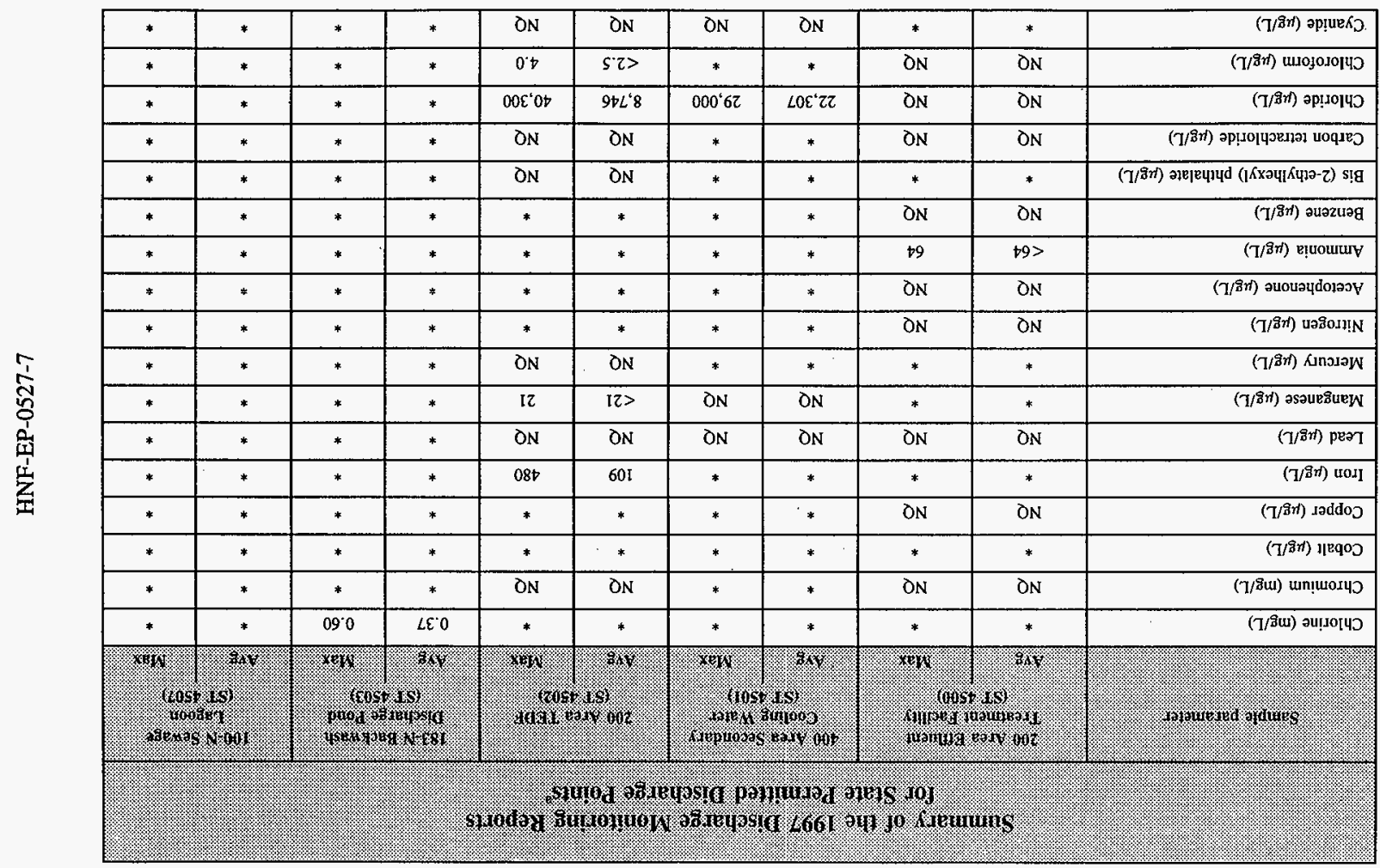




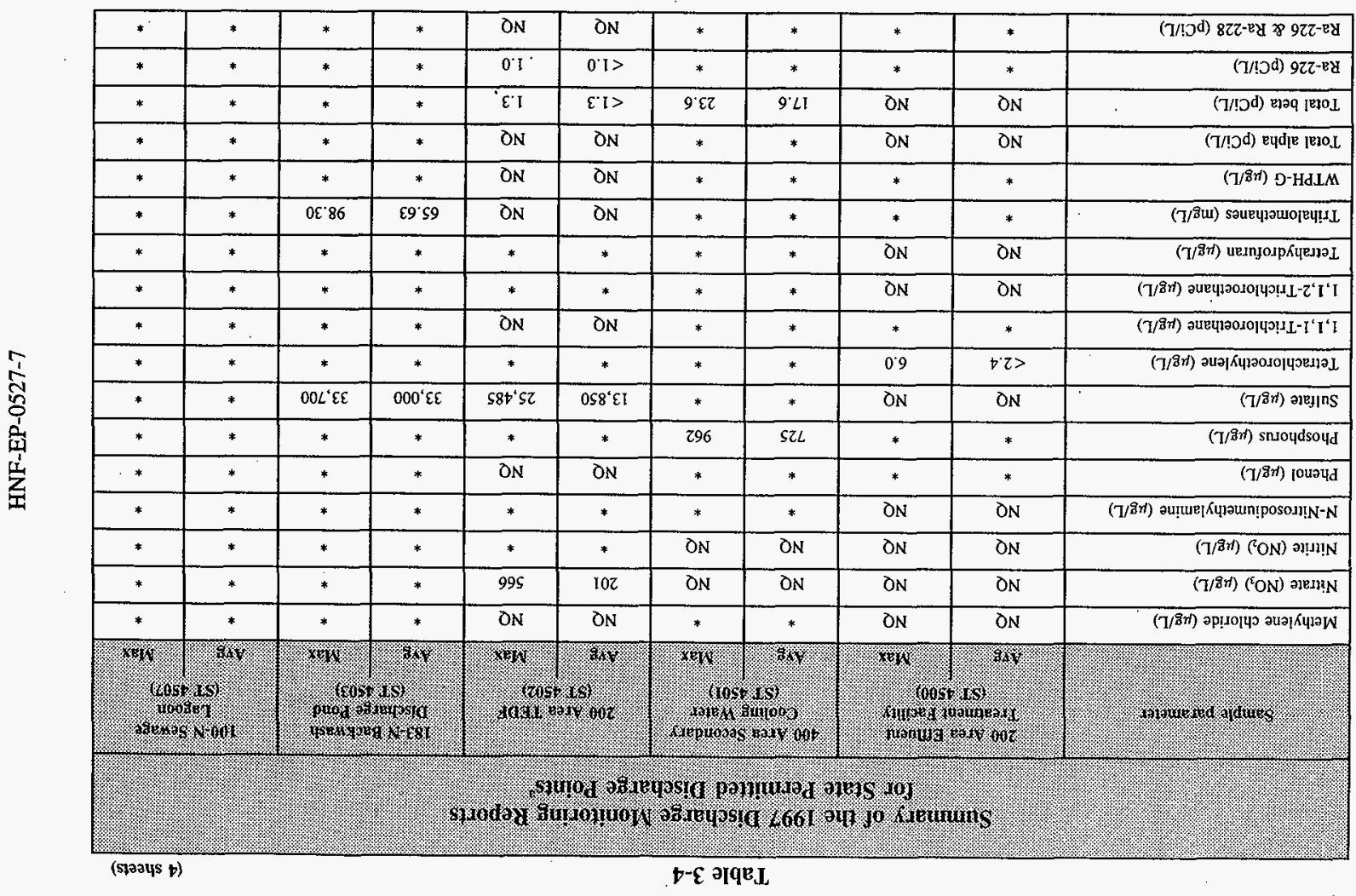




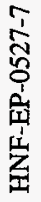

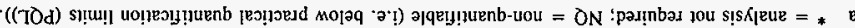

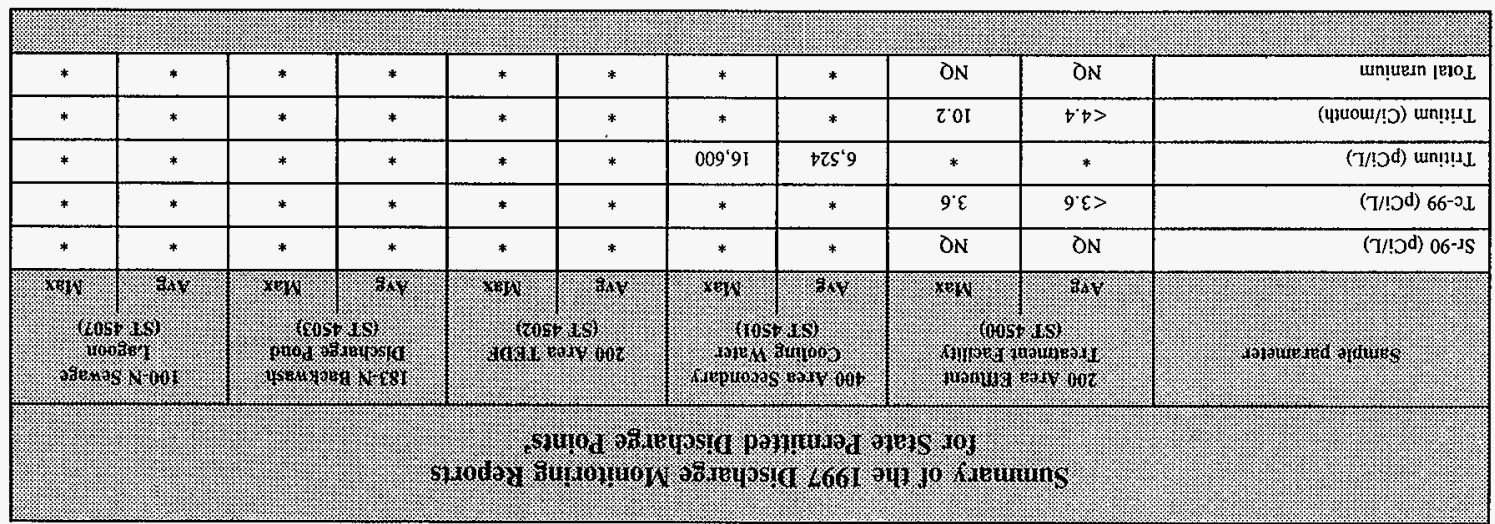


Table 3-5

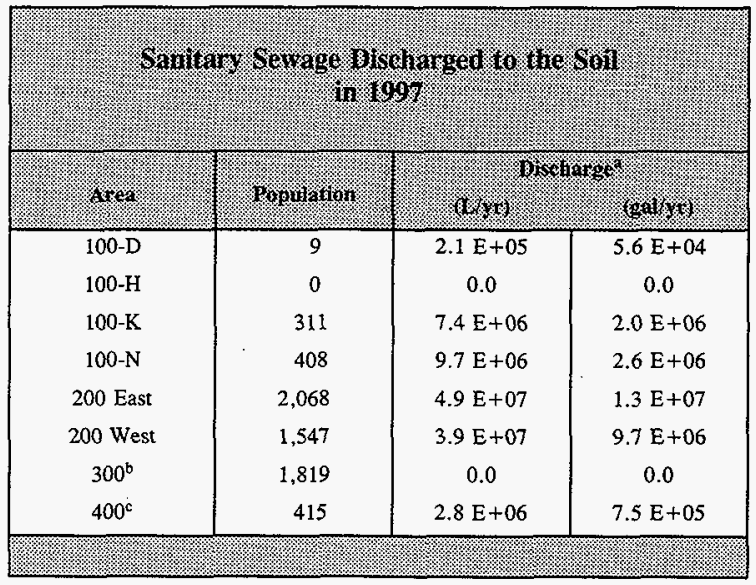

Notes:

a Discharges estimated by multiplying the total number of persons assigned to each area by $95 \mathrm{~L} /$ day-person ( 25 gal/day-person) and by 251 business days.

b Discharges from the 300 Area no longer go to the soil column.

c Discharges from the 400 Area were discontinued on April 15, 1998. 
HNF-EP-0527-7

This page intentionally left blank. 


\subsection{HAZARDOUS SUBSTANCE RELEASES}

A hazardous substance released to the environment is required to be evaluated to determine if it is reportable to the appropriate federal, state, and local regulatory agency(s). If the quantity released meets or exceeds the reporting thresholds, the notification is required. Reportable releases of hazardous substances are classified as the following two types:

- Nonroutine releases

- Continuous, routine releases.

Information for each type of release is discussed in the following sections.

\subsection{NONROUTINE RELEASES}

The following listing shows the number of non-routine releases of a solid, semi-solid, liquid, or airborne substance involving radioactive, hazardous, or dangerous wastes, hazardous or extremely hazardous substances, polychlorinated biphenyls (PCBs), and oil and/or petroleum derivatives for 1997 .

\section{Fluor Daniel Hanford}

- 7 releases were evaluated and determined to be reportable and 242 other releases were evaluated and determined to be nonreportable per regulatory notification requirements for FDH and its subcontractors

Bechtel Hanford, Incorporated

- 0 releases were evaluated and determined to be reportable and 79 other releases were evaluated and determined to be nonreportable per regulatory notification requirements for $\mathrm{BHI}$ and its subcontractors

\subsection{ROUTINE CONTINUOUS RELEASES}

Releases of hazardous substances that exceed CERCLA reportable quantities (RQ) need not be reported immediately to the National Response Center when both of the following conditions are met:

- An initial notification has been completed

- The routine releases are continuous and stable in quantity and rate. 
The initial notification requirement has been satisfied concerning hazardous substances that have exceeded or have a potential to exceed an RQ. Historically only the continuous routine releases of ammonia, ammonium hydroxide, and carbon tetrachloride have posed operational difficulties in staying beneath RQs. For 1997, releases of ammonia, ammonium hydroxide, and carbon tetrachloride were below reportable quantities and were continuous and stable in quantity and rate. 
HNF-EP-0527-7

\subsection{REFERENCES}

DOE Order 5400.1, General Environmental Protection Program.

DOE Order 5400.5, Radiation Protection of the Public and the Environment.

DOE Order 5480.1B, Environment, Safety, and Health Program for Department of Energy Operations.

DOE/RL-98-33, Radionuclide Air Emissions Report for the Hanford Site, Calendar Year 1997, U.S. Department of Energy, Richland Field Office, Richland, Washington.

EPA 450/4-90-003, AIRS Facility Subsystem Source Classification Codes and Emission Factor Listing Criteria Air Pollutants, U.S. Environmental Protection Agency, Research Triangle Park, North Carolina.

HNF-EP-0573-6, Hanford Site Near-Facility Environmental Monitoring Annual Report, Calendar Year 1997, Waste Management Federal Services, Inc., Northwest Operations, Richland, Washington.

PNNL-11795, Hanford Site Environmental Report for Calendar Year 1998, Pacific Northwest National Laboratory, Richland, Washington.

PNNL-11796, 1997 Surface Environmental Surveillance Data, Pacific Northwest National Laboratory, Richland, Washington.

ST 4500, State Waste Discharge Permit, 200 Area Effluent Treatment Facility, Washington State Department of Ecology,. June 26, 1995, Olympia, Washington.

ST 4501, State Waste Discharge Permit, 400 Area Secondary Cooling Water, Washington State Department of Ecology, July 31, 1996, Olympia, Washington.

ST 4502, State Waste Discharge Permit, 200 Area Treated Effluent Disposal Facility, Washington State Department of Ecology, April 18, 1995, Olympia, Washington.

ST 4503, State Waste Discharge Permit, 183-N Backwash Discharge Pond, Washington State Department of Ecology, May 12, 1997, Olympia, Washington.

ST 4507, State Waste Discharge Permit, 100-N Sewage Treatment Lagoon, Washington State Department of Ecology, May 12, 1997, Olympia, Washington.

ST 4508, State Waste Discharge Permit, Hydrotest, Maintenance, and Construction Discharges, Washington State Department of Ecology, May 30, 1998, Olympia, Washington. 
ST 4509, State Waste Discharge Permit, Cooling Water and Condensate Discharges, Washington State Department of Ecology, May 1, 1998, Olympia, Washington.

WA-000374-3, Authorization to Discharge Under the National Pollutant Discharge Elimination System, Discharges from Facilities on the Hanford Reservation to the Columbia River, U.S. Environmental Protection Agency Region 10, December 7, 1981, Seattle, Washington.

WA-002591-7, Authorization to Discharge Under the National Pollutant Discharge Elimination System, Discharges from the 300 Area Treated Effluent Disposal Facility to the Columbia River, U.S. Environmental Protection Agency Region 10, October 31,1994 , Seattle, Washington.

WA-R-10-000F, Authorization to Discharge Under the National Pollutant Discharge Elimination System, Storm Water Discharges from Construction Activities that are Classified as Associated with Industrial Activity, U.S. Environmental Protection Agency Region 10, September 9, 1992, Seattle, Washington. 


\section{DISTRIBUTION}

Number of Copies

$\underline{\text { OFFSITE }}$

U.S. Environmental Protection Agency, Region 10 1200 Sixth Avenue

Seattle, WA 98101

J. M. Leitch (5)

AT-081

A. Frankel

State of Washington. Department of Health

Air Emissions and Defense Waste Section

Division of Radiation Protection

P. O. Box 47827

Olympia, WA 98504-7827
A. W. Conklin (5)

J. E. Erickson

1

State of Washington, Department of Ecology

M. A. Wilson

B5-18

1

U.S. Department of Energy-Headquarters

K. C. Duvall

EH-232

1

Confederated Tribes of the Umatilla Indian Reservation

P. O. Box 638

Pendleton, OR 97801

J. R. Wilkinson

Nez Perce Tribe

Environmental Restoration and Waste Management

P. O. Box 365

Lapwai, ID 83540

D. L. Powaukee 


\section{DISTRIBUTION (continued)}

\section{Number of Copies}

\section{OFFSITE}

1

\section{Yakama Indian Nation}

Environmental Restoration Waste Management Program P.O. Box 151

Toppenish, Washington 98948

R. Jim

\section{ONSITE}

11

U.S. Department of Energy

Richland Operations Office

G. M. Bell

A5-52

R. F. Brich

HO-12

S. E. Clarke

A5-15

J. B. Hall

A5-15

J. E. Mecca

R3-81

B. M. Pangbom

A5-55

J. E. Rasmussen

A5-15

H. M. Rodriguez

A5-15

D. C. Ward

A5-15

A. H. Wirkkala

A5-11

DOE-RL Public Reading Room

B\&W Hanford Company

J. M. Barnett

L1-05

T. G. Beam

S6-51

J. E. Bramson

T5-54

B. C. Cornwell

L6-26

N. R. Dahl

$\mathrm{N} 2-57$

T. A. Dillhoff

N2-57

D. L. Johnson

S6-01

G. J. LeBaron

S6-19

D. E. Rasmussen

N1-47

D. L. Wiegand

T5-54

C. D. Wollam

S6-22 


\section{DISTRIBUTION (continued)}

Bechtel Hanford, Inc.

R. G. Egge

M. E. Greenidge

R. J. Landon

J. J. McGuire

HO- 02

D. W. Long

\$3-20

M. R. Morton

X1-86

J. G. Woolard

HO-18

J. P. Zoric

HO-02

G. J. Carter, Jr.

CH2M Hill Ine.

E. T. Coenenberg

H9-03

S. J. Ingle

H9-01

2

DE\&S Hanford, Inc.

R. G. Gant

X3-79

D. J. Watson

$\times 3-79$

DynCorp Tri-Cities Services, Inc.

M. J. Brown

G3̈-08

B. J. Dixon

G3-26

C. E. Marple

N1-23

Fluor Daniel Hanford. Inc.

President's Office

H5-20

W. D. Adair

J. A. Bates

H6-21

J. J. Kapadia

H6-23

C. G. Mattsson

H6-23

N1-26

K. A. Peterson

H6-23

S. M. Price

H6-23

D. G. Ranade

H6-23

W. E. Toebe

H6-23

B. D. Williamson

B3-15 


\section{DISTRIBUTION (continued)}

Lockheed Martin Hanford Corporation

M. S. Allen

D. J. Carrell

G. M. Crummel

R1-51

D. L. Dyekman

S7-03

K. A. Elsethagen

S5-03

B. G. Erlandson

R1-51

P. C. Miller

R1-51

1

Lockheed Martin Services, Inc.

Central Files

DPC

Numatec Hanford Corporation

R. A. Kaldor

H5-25

7

Pacific Northwest National Laboratory

R. L. Dirkes

K6-75

W. T. Farris

K3-54

R. W. Hanf, Jr.

K6-75

G. R. Hoenes

P7-79

J. P. McDonald

K6-96

K. Rhoads

K3-54

PNNL Reference Library

P8-55

Waste Management Federal Services of Hanford, Inc.

B. M. Barnes

T4-04

R. J. Boom

T6-12

M. W. Bowman

S6-72

H. C. Boynton

T4-52

J. R. Buckley

T3-04

B. L. Curn

H6-36

W. E. Davis

H6-36

L. P. Diediker (75)

H6-36

D. L. Flyckt

S6-71

T. P. Frazier

H6-25 
DISTRIBUTYON (continued)

Waste Management Federal Services of Hanford, Inc. (continued)

B. P. Gleckler

W. E. Green

H6-36

E. M. Greager

H6-36

M. D. Guthrie

H6-36

R. D. Haggard

S6-72

D. L. Halgren

H6-25

J. S. Hill

L6-04

N. A. Homan

H6-25

R. E. Johnson

H6-25

L. D. Kamberg

H6-25

J. J. Luke

H6-25

J. A. Morrison

H6-25

J. K. Perry

S3-25

D. L. Renberger

H6-25

R. W. Szelmeczka

T3-03

D. B. Van Leuven

L6-05

G. T. Wells

H6-10

J. A. Winterhalder

H6-36

Correspondence Control

H6-21

A3-01

Waste Management Federal Services, Inc.

Northwest Operations

J. J. Dorian

H1-13

D. L. Edwards

H1-12

A. R. Johnson

H1-13

B. M. Markes

H1-13

S. M. McKinney

H1-12

C. J. Perkins

$\mathrm{H} 1-12$ 
HNF-EP-0527-7

This page intentionally left blank. 